\title{
Semidiscrete and Single Step Fully Discrete Approximations for Second Order Hyperbolic Equations With Time-Dependent Coefficients
}

\author{
By Laurence A. Bales
}

\begin{abstract}
L^{2}$ norm error estimates are proved for finite element approximations to the solutions of initial boundary value problems for second order hyperbolic partial differential equations with time-dependent coefficients. Optimal order rates of convergence are shown for semidiscrete and single step fully discrete schemes using specially constructed initial data. The initial data are designed so that the data used for the fully discrete equation is reasonable to compute and so that the optimal order estimates can be proved.
\end{abstract}

1. Introduction. In this paper we shall consider the approximate solution of the initial boundary value problem

$$
\begin{array}{cl}
u_{t t}=-L(t) u \equiv \sum_{j, k=1}^{N} \frac{\partial}{\partial x_{j}}\left(a_{j k}(x, t) \frac{\partial u}{\partial x_{k}}\right)-a_{0}(x, t) u & \text { in } \Omega \times(0, \tau], \\
u(x, t)=0 & \text { on } \partial \Omega \times(0, \tau] \\
u(x, 0)=u^{0}(x) & \text { in } \Omega, \\
u_{t}(x, 0)=u_{t}^{0}(x) & \text { in } \Omega .
\end{array}
$$

Here $\Omega$ is a bounded domain in $\mathbf{R}^{N}$ with sufficiently smooth boundary $\partial \Omega, a_{j k}(x, t)$ and $a_{0}(x, t)$ are sufficiently smooth real-valued functions, the matrix $\left(a_{j k}\right)$ is symmetric and uniformly positive definite and $a_{0}$ is nonnegative on $\bar{\Omega}$.

For $s \geqslant 0, H^{s}(\Omega)=H^{s}$ will denote the Sobolev space of real-valued functions on $\Omega$. The norm on $H^{s}$ is denoted by $\|\cdot\|_{s}$. The inner product on $L^{2}(\Omega)=L^{2}=H^{0}$ is denoted by $(\cdot, \cdot)$ and the associated norm by $\|\cdot\| . H_{0}^{1}$ denotes the subspace of functions in $H^{1}$ that vanish (in the sense of trace) on $\partial \Omega$.

$L(t)$ is a smooth family of unbounded selfadjoint operators on $L^{2}$ with domain $D_{L}=H^{2} \cap H_{0}^{1}$ and a smooth family of bounded operators from $H^{l+2} \cap D_{L}$ to $H^{l}$, for $l \geqslant 0 . L^{(j)}(t)=(d / d t)^{j} L(t)$ is calculated by differentiating the coefficients of $L(t)$ with respect to time.

The solution operator $T(t): L^{2} \rightarrow D_{L}$ of the elliptic problem $(w=T f)$

$$
L w=f \quad \text { in } \Omega, \quad w=0 \text { on } \partial \Omega,
$$

satisfies the equation

$$
a(T(t) f, v)=(f, v) \text { for all } v \in H_{0}^{1},
$$

Received August 3, 1983; revised December 16, 1983.

1980 Mathematics Subject Classification. Primary 65M15; Secondary 65N30. 
for given $f \in L^{2}$, where $a(\cdot, \cdot)$ is the bilinear form

$$
a(\phi, \psi)=\int_{\Omega}\left(\sum_{j, k=1}^{N} a_{j k} \frac{\partial \phi}{\partial x_{j}} \frac{\partial \psi}{\partial x_{k}}+a_{0} \phi \psi\right) d x
$$

for $\phi, \psi \in H^{1}$. For $l \geqslant 0, T(t)$ is a smooth family of bounded operators from $H^{l}$ to $H^{l+2} \cap D_{L}$. For $m \geqslant 0$, the bilinear form $a^{(m)}(\cdot, \cdot)$ is defined as

$$
a^{(m)}(\phi, \psi) \equiv \int_{\Omega}\left(\sum_{j, k=1}^{N} \frac{\partial^{m} a_{j k}}{\partial t^{m}} \frac{\partial \phi}{\partial x_{j}} \frac{\partial \psi}{\partial x_{k}}+\frac{\partial^{m} a_{0}}{\partial t^{m}} \phi \psi\right) d x
$$

for $\phi, \psi \in H^{1}$.

Section 2 contains two regularity theorems for the solution of (1.1).

In Section 3, the approximate solution operator $T_{h}$ (defined using a Galerkin finite element method on subspaces of piecewise polynomials of degree $r-1$ ) is introduced and is used to define the semidiscrete approximation $u_{h}(t)$. For integer $J \geqslant 0$, the following estimate is proved.

$$
\begin{aligned}
\sup _{0 \leqslant t \leqslant \tau} & \left\|\left(u^{(J)}(t)-u_{h}^{(J)}(t)\right)\right\| \\
\leqslant & C h^{r}\left(\left\|u^{0}\right\|_{J+r+1}+\left\|u_{t}^{0}\right\|_{J+r}\right) \\
& \quad+C \sum_{j=0}^{J}\left(\left\|T_{h}^{1 / 2}(0)\left(u^{(j+1)}(0)-u_{h}^{(j+1)}(0)\right)\right\|+\left\|u^{(j)}(0)-u_{h}^{(j)}(0)\right\|\right) .
\end{aligned}
$$

Here $u^{(j)}(t)$ and $u_{h}^{(j)}(t)$, for $j=0, \ldots, J$, denote the $j$ th time derivative of $u(t)$ and $u_{h}(t)$, respectively.

In Section 4, a single step fully discrete approximation $W_{1}^{n}$ to $u_{h}(n k)$, where $k$ denotes the discrete time step, is defined. These schemes are based on a class of methods for approximately solving stiff ordinary differential equations. Methods of order $\nu$ are considered, where $1 \leqslant \nu \leqslant 4$. The fourth order method corresponds to the 2-2 rational Padé approximation to the exponential when it is applied to equations with time-independent coefficients. Only unconditionally stable (e.g., $A$ stable methods) are considered. The following fully discrete error estimate is proved:

$$
\begin{aligned}
\left\|u_{h}(\tau)-W_{1}^{N}\right\| \leqslant & C k^{\nu} \sum_{j=0}^{\nu+1}\left(\left\|T_{h}^{1 / 2}(0) u_{h}^{(j+1)}(0)\right\|+\left\|u_{h}^{(j)}(0)\right\|\right) \\
& +C\left\|Q_{0}\left(U_{h}(0)-W^{0}\right)\right\| \|_{0}
\end{aligned}
$$

where $N=\tau / k$ and $\left\|Q_{0}\left(U_{h}(0)-W^{0}\right)\right\|_{0}$ measures the error in the initial data of the fully discrete problem.

In Section 5, special operators are constructed and are used to define the initial data $U_{h}(0)$ for the semidiscrete problem. $U_{h}(0)$ never has to be computed since it is close to a special choice of the initial data $W^{0}$ (which is reasonable to compute) for the fully discrete problem. The proofs of Theorems 5.1 and 5.2 are contained in Section 8 which appears in the supplements section of this issue.

In Section 6, the results of Sections 3, 4 and 5 are combined in order to derive the $L^{2}$ error estimates. The special definitions of $U_{h}(0)$ and $W^{0}$ imply appropriate bounds for the terms after the inequalities in (1.5) and (1.6). In other words, optimal 
order error estimates for the initial data of the semidiscrete and fully discrete problems and bounds for time derivatives of the solution of the semidiscrete equation are obtained. Therefore, the triangle inequality and (1.5) and (1.6) imply the optimal $L^{2}$ estimate

$$
\left\|u(\tau)-W_{1}^{N}\right\| \leqslant C\left(h^{r}+k^{\nu}\right)\left(\left\|u^{0}\right\|_{p}+\left\|u_{t}^{0}\right\|_{p-1}\right)
$$

for a certain integer $p$.

There is an extensive literature concerned with the error analysis of the methods mentioned above for linear parabolic equations and second order linear hyperbolic equations. Bramble, Schatz, Thomée and Wahlbin [6] analyzed semidiscrete approximations for parabolic equations with time-independent coefficients and Baker, Bramble, and Thomée [3] analyzed single step fully discrete approximations. Zlámal [22] analyzed fully discrete approximations based on linear multi-step methods. Sammon in [20] and [21], Bramble and Sammon [5] and Mingyou and Thomée [19] analyzed approximations for parabolic equations with time-dependent coefficients.

For second order hyperbolic equations with time-independent coefficients Baker and Bramble [2] analyzed semidiscrete and single step fully discrete approximations. Baker, Dougalis and Serbin [4] analyzed fully discrete approximations based on two-step schemes. Two-step schemes producing second order accuracy were also studied by Baker [1] and Dupont [11]. In addition, for high order single step methods, see Crouzeix [7] and Gekeler [13], and for multi-step methods, see Dougalis [8] and Gekeler [12].

Some of the techniques and results in this work are similar to those in Bramble and Sammon [5], Sammon [20], [21], where parabolic equations with time-dependent coefficients were analyzed. The analysis here for the hyperbolic equation (1.1) involves noncommutative skew-symmetric operators (the analogous operators in the parabolic case are selfadjoint and positive definite) and special time-dependent inner products.

Throughout this work $C$ (sometimes used with a subscript) will denote a general positive constant which is not necessarily the same in any two places.

2. Regularity. This section contains two regularity theorems for the solution $u$ of (1.1) in $L^{2}$-based Sobolev spaces. Theorem 2.1 concerns time derivatives of $u$ and Theorem 2.2 includes estimates for mixed (time and space) derivatives of $u$.

Let $C^{0}([0, \tau] ; H)$ be the space of continuous functions from $[0, \tau]$ to the Hilbert space $H$ with the norm

$$
\|f\|_{C^{0}([0, \tau]: H)}=\sup _{t \in[0, \tau]}\|f(t)\|_{H}<+\infty
$$

For integer $i \geqslant 0$ let $u^{(i)}$ denote the $i$ th time derivative of $u$. Also, define

$$
\begin{aligned}
& u_{0}=u^{0}, \\
& u_{1}=u_{t}^{0}, \text { and } \\
& u_{i}=\sum_{j=0}^{i-2}\left(\begin{array}{c}
i-2 \\
j
\end{array}\right) L^{(i-2-j)}(0) u_{j} \quad \text { for } i \geqslant 2
\end{aligned}
$$


$u_{i}$ is defined to correspond to the $i$ th time derivative of $u$ at $t=0$ and for $i \geqslant 2$ is obtained by formally differentiating the equation $u_{t t}=-L u i-2$ times. Note that for $i \geqslant 2$

$$
\left\|u_{i}\right\| \leqslant C\left(\left\|u^{0}\right\|_{i}+\left\|u_{t}^{0}\right\|_{i-1}\right) \text { and }\left\|u_{i-1}\right\|_{1} \leqslant C\left(\left\|u^{0}\right\|_{i}+\left\|u_{t}^{0}\right\|_{i-1}\right) .
$$

THEOREM 2.1. Let $i$ be a nonnegative integer. If

$$
u_{j} \in D_{L} \text { for } j=0, \ldots, i \text { and } u_{i+1} \in H_{0}^{1},
$$

then there is a unique function $u$ such that, for $t \in[0, \tau], u(t) \in H_{0}^{1}$ and

$$
\begin{aligned}
& \left(u_{t t}, \phi\right)+a(u, \phi)=0 \quad \text { for all } \phi \in H_{0}^{1}, \\
& u(0)=u^{0} \quad \text { in } H_{0}^{1}, \quad \text { and } \\
& u_{t}(0)=u_{t}^{0} \quad \text { in } L^{2} .
\end{aligned}
$$

Furthermore,

$$
\begin{gathered}
\left\|u^{(i)}\right\| C^{0}\left([0, \tau] ; D_{L}\right)+\left\|u^{(i+1)}\right\| C^{0}\left([0, \tau] ; H_{0}^{1}\right)+\left\|u^{(i+2)}\right\| C^{0}\left([0, \tau] ; L^{2}\right) \\
\leqslant C\left(\left\|u^{0}\right\|_{i+2}+\left\|u_{t}^{0}\right\|_{i+1}\right)
\end{gathered}
$$

and

$$
u^{(i+2)}+L u^{(i)}+\sum_{j=0}^{i-1}\left(\begin{array}{l}
i \\
j
\end{array}\right) L^{(i-j)} u^{(j)}=0 \quad \text { in } C^{0}\left([0, \tau] ; L^{2}\right)
$$

Proof. Notice that (2.3) implies that $u_{i+2} \in L^{2}$. From Gilardi [14] the solution of (2.4) exists and is unique and

$$
\left\|u^{(i+2)}\right\| C^{0}\left([0, \tau] ; L^{2}\right)+\sum_{j=0}^{i+1}\left\|u^{(j)}\right\| C^{0}\left([0, \tau] ; H_{0}^{1}\right) \leqslant C\left(\sum_{j=0}^{i+1}\left\|u_{j}\right\|_{1}+\sum_{j=0}^{i+2}\left\|u_{j}\right\|\right) .
$$

Let $\phi \in H_{0}^{1}$. Differentiating the equation

$$
\left(u^{(2)}(t), \phi\right)+a(u(t), \phi)=0
$$

$k$ times gives

$$
\left(u^{(k+2)}(t), \phi\right)+a\left(u^{(k)}(t), \phi\right)+\sum_{j=0}^{k-1}\left(\begin{array}{l}
k \\
j
\end{array}\right) a^{(k-j)}\left(u^{(j)}(t), \phi\right)=0 .
$$

The remaining parts of the theorem are proved by induction on $k$. For $k=0$ we have

$$
\left(u^{(2)}(t), \phi\right)+a(u(t), \phi)=0 .
$$

Since $u^{(2)}(t) \in L^{2}$, by elliptic regularity

$$
u(t) \in D_{L} \quad \text { and } \quad u^{(2)}(t)+L(t) u(t)=0 .
$$

Furthermore,

$$
\begin{aligned}
& C\|u(t+h)-u(t)\|_{2} \leqslant\|L(t+h)(u(t+h)-u(t))\| \\
& \leqslant\|L(t+h) u(t+h)-L(t) u(t)\|+\|L(t) u(t)-L(t+h) u(t)\| \\
& \leqslant\left\|u^{(2)}(t+h)-u^{(2)}(t)\right\|+C h\|u(t)\|_{2} .
\end{aligned}
$$


Since $u^{(2)} \in C^{0}\left([0, \tau] ; L^{2}\right)$ and $u(t) \in D_{L}$, it follows that $u \in C^{0}\left([0, \tau] ; D_{L}\right)$ and $\|u\|_{C^{0}\left([0, \tau] ; D_{L}\right)} \leqslant C\left\|u^{(2)}\right\|_{C^{0}\left([0, \tau] ; L^{2}\right)}$. Now assume

$$
\left\|u^{(j)}\right\| C_{C^{0}\left([0, \tau] ; D_{L}\right)} \leqslant C \sum_{p=0}^{j+2}\left\|u^{(p)}\right\|_{C^{0}\left([0, \tau] ; L^{2}\right)} \quad \text { for } j=0, \ldots, k-1 \text { and } k \leqslant i .
$$

Integrating by parts in (2.8) gives

$$
\left(u^{(k+2)}(t), \phi\right)+a\left(u^{(k)}(t), \phi\right)+\sum_{j=0}^{k-1}\left(\begin{array}{l}
k \\
j
\end{array}\right)\left(L^{(k-j)} u^{(j)}(t), \phi\right)=0 .
$$

An argument similar to the one given above for $k=0$ gives

$$
u^{(k+2)}(t)+L u^{(k)}(t)+\sum_{j=0}^{k-1}\left(\begin{array}{l}
k \\
j
\end{array}\right) L^{(k-j)}(t) u^{(j)}(t)=0
$$

and $u^{(k)} \in C^{0}\left([0, \tau] ; D_{L}\right)$. Also,

$$
\left\|\boldsymbol{u}^{(k)}\right\|_{C^{0}\left([0, \tau] ; D_{L}\right)} \leqslant C\left(\sum_{j=0}^{k+2}\left\|\boldsymbol{u}^{(j)}\right\|_{i C^{0}\left([0, \tau] ; L^{2}\right)}\right) .
$$

Combining (2.2), (2.7) and (2.9) proves the theorem.

The following technical lemma will be used in the proof of Theorem 2.2.

LEMMA 2.1. For integers $p \geqslant 1$ and $l \geqslant 0$, the operator

$$
T^{(p)}=-\sum_{j=0}^{p-1}\left(\begin{array}{c}
p \\
j
\end{array}\right) T L^{(p-j)} T^{(j)}
$$

is a bounded operator from $H^{l}$ to $H^{l+2} \cap H_{0}^{1}$.

Proof. Since

$$
\begin{aligned}
\left\|T^{(p)} f\right\|_{l+2} & \leqslant C \sum_{j=0}^{p-1}\left\|T L^{(p-j)} T^{(j)}\right\|_{l+2} \\
& \leqslant C \sum_{j=0}^{p-1}\left\|L^{(p-j)} T^{(j)} f\right\|_{l} \leqslant C \sum_{j=0}^{p-1}\left\|T^{(j)} f\right\|_{l+2},
\end{aligned}
$$

and $T$ is a bounded operator from $H^{l}$ to $H^{l+2} \cap H_{0}^{1}$, the result follows by induction.

We can now prove

THEOREM 2.2. For integers $p \geqslant 0$ and $m \geqslant 1$, the solution $u$ of (1.1) satisfies the following estimates for $t \in[0, \tau]$ :

$$
\begin{gathered}
\left\|\boldsymbol{u}^{(p)}(t)\right\|_{2 m} \leqslant C \sum_{j=2}^{p+2 m}\left\|\boldsymbol{u}^{(j)}(t)\right\|, \\
\left\|\boldsymbol{u}^{(p+1)}(t)\right\|_{2 m-1} \leqslant C \sum_{j=1}^{p+2 m-1}\left\|\boldsymbol{u}^{(j)}(t)\right\|_{1} .
\end{gathered}
$$

Proof. Equation (1.1) can be written $u=-T u_{t t}$, so that

$$
u^{(p)}=-\sum_{j=0}^{p}\left(\begin{array}{l}
p \\
j
\end{array}\right) T^{(p-j)} u^{(j+2)} .
$$


Therefore,

$$
\left\|u^{(p)}\right\|_{2 m} \leqslant C \sum_{j=0}^{p}\left\|T^{(p-j)} u^{(j+2)}\right\|_{2 m} .
$$

Lemma 2.1 and (2.13) imply that

$$
\left\|u^{(p)}\right\|_{2 m} \leqslant C \sum_{j=0}^{p}\left\|u^{(j+2)}\right\|_{2 m-2}=C \sum_{j=2}^{p+2}\left\|u^{(j)}\right\|_{2 m-2} .
$$

(2.11) follows from (2.14) by induction. The proof of (2.12) is similar.

Note that Theorem 2.1 and Theorem 2.2 imply that if $u_{j} \in D_{L}$ for $j=0,1, \ldots$, $p+m-2$ and $u_{p+m-1} \in H_{0}^{1}$, where $p \geqslant 0$ and $m \geqslant 0$ are integers such that $p+m \geqslant 2$, then for $t \in[0, \tau]$

$$
\left\|u^{(p)}(t)\right\|_{m} \leqslant C\left(\left\|u^{0}\right\|_{p+m}+\left\|u_{t}^{0}\right\|_{p+m-1}\right) .
$$

3. Semidiscrete Approximations. The solution operator $T$ of the associated elliptic boundary value problem which is defined by

$$
a(T f, v)=(f, v) \text { for all } v \in H_{0}^{1} \text {, for given } f \in L^{2},
$$

satisfies

$$
T L=I \quad \text { on } D_{L} \quad \text { and } \quad L T=I \quad \text { on } L^{2} .
$$

Let $0<h<1$ be a parameter, and $\left\{S_{h}\right\}_{0<h<1}$ a family of finite dimensional subspaces of $L^{2}$. We shall assume that we are given a corresponding family of operators $T_{h}: L^{2} \rightarrow S_{h}$ which approximates $T$ and has the following properties:

(i) $T_{h}$ is selfadjoint, positive semidefinite on $L^{2}$, and positive definite on $\dot{S}_{h}$.

(ii) There is a positive integer $r \geqslant 2$, such that for integer $j \geqslant 0$, there exists a constant $C(j)$ with

$$
\left\|\left(T^{(j)}-T_{h}^{(j)}\right) f\right\| \leqslant C(j) h^{s}\|f\|_{s-2}
$$

for all $f \in H^{s-2}, 2 \leqslant s \leqslant r$, and where $T^{(j)}$ and $T_{h}^{(j)}$ denote the $j$ th time derivative of $T$ and $T_{h}$, respectively.

(iii) On $S_{h}$ define $L_{h}(t)=\left(T_{h}(t)\right)^{-1}$ and $L_{h}^{(k)}(t)=d^{k} L_{h}(t) / d t^{k}$. For integer $k \geqslant 0$, there exists a constant $C=C(k)$, which is independent of $h$, such that for $t, s \in[0, \tau]$

$$
\left|\left(L_{h}^{(k)}(t) \phi, \phi\right)\right| \leqslant C(k)\left(L_{h}(s) \phi, \phi\right) \quad \text { for all } \phi \in S_{h} \text {. }
$$

An example of a family of operators satisfying these assumptions is given by the following: Suppose that $S_{h} \subset H_{0}^{1}$ so that elements of $S_{h}$ vanish on $\partial \Omega$. Assume further that $S_{h}$ has the approximation property

$$
\inf _{\chi \in S_{h}}\left\{\|w-\chi\|+h\|w-\chi\|_{1}\right\} \leqslant c h^{s}\|w\|_{s} \quad \text { for } 1 \leqslant s \leqslant r .
$$

The operators $T_{h}: L^{2} \rightarrow S_{h}$ are defined by

$$
a\left(T_{h} f, \chi\right)=(f, \chi) \text { for all } \chi \in S_{h} \text {, for given } f \in L^{2} .
$$

With $T_{h}$ defined by (3.3) it follows that

$$
\left(L_{h} \phi, \psi\right)=a(\phi, \psi) \text { for all } \phi, \psi \in S_{h} .
$$


For more details and other examples see Bramble, Schatz, Thomée and Wahlbin [6], Baker and Bramble [2], Mingyou and Thomée [19], Sammon [21], and Section 7.

The hyperbolic problem (1.1) can be written

$$
T u_{t t}+u=0, \quad u(0)=u^{0}, \quad u_{t}(0)=u_{t}^{0} .
$$

The semidiscrete approximation for the solution $u$ is defined as the mapping $u_{h}$ : $[0, \tau] \rightarrow S_{h}$ satisfying

$$
\begin{aligned}
& T_{h}\left(u_{h}\right)_{t t}+u_{h}=0, \quad 0 \leqslant t \leqslant \tau, \\
& u_{h}(0)=v^{0}, \quad\left(u_{h}\right)_{t}(0)=v_{t}^{0},
\end{aligned}
$$

where $v^{0}$ and $v_{t}^{0}$ are elements of $S_{h}$ which will be chosen to be close to $u^{0}$ and $u_{t}^{0}$.

This section contains two theorems about time derivatives of $u_{h}$. Theorem 3.1 is a bound for time derivatives of $u_{h}(t)$ in terms of time derivatives at $t=0$. Theorem 3.2 is a bound for time derivatives of $u(t)-u_{h}(t)$ in terms of its time derivatives at $t=0$ and truncation error. The bounds derived for time derivatives of $u(t)-u_{h}(t)$ are used with a special choice of the initial data $u_{h}(0)$ and $\left(u_{h}\right)_{t}(0)$. In Section 6 , we will show how this special choice for the initial data for the semidiscrete equation fits perfectly into the analysis of the fully discrete approximation. These two theorems concerning bounds for time derivatives of $u_{h}$ are proved using energy methods.

Differentiation of (3.4) yields terms containing time derivatives of $T_{h}$. In order to bound these terms in the energy estimates we prove a technical lemma. Since $T_{h} L_{h}=L_{h} T_{h}=I$ on $S_{h}$, for $k>0$

$$
\frac{d^{k}}{d t^{k}}\left(T_{h} L_{h}\right)=\sum_{j=0}^{k}\left(\begin{array}{l}
k \\
j
\end{array}\right) T_{h}^{(k-j)} L_{h}^{(j)}=0
$$

and

$$
\frac{d^{k}}{d t^{k}}\left(L_{h} T_{h}\right)=\sum_{j=0}^{k}\left(\begin{array}{l}
k \\
j
\end{array}\right) L_{h}^{(k-j)} T_{h}^{(j)}=0 .
$$

Similar equations are valid (on the appropriate function spaces) if $T_{h}$ and $L_{h}$ are replaced by $T$ and $L$, respectively. Since $T_{h}$ and $L_{h}$ are positive definite on $S_{h}, T_{h}^{1 / 2}$ and $L_{h}^{1 / 2}$ exist and are positive definite on $S_{h}$. We note that (3.3) implies that $T_{h} P=T_{h}$, where $P$ is the orthogonal projection in $L^{2}$ from $L^{2}$ to $S_{h}$.

Lemma 3.1. Let $k \geqslant 0$ be an integer and $s, t \in[0, \tau]$. Then for all $\phi \in S_{h}$, there exists a constant $C$ which is independent of $h$ such that

$$
\left\|T_{h}^{1 / 2}(s) L_{h}^{(k)}(t) T_{h}^{1 / 2}(s) \phi\right\| \leqslant C\|\phi\|
$$

and

$$
\left\|L_{h}^{1 / 2}(s) T_{h}^{(k)}(t) L_{h}^{1 / 2}(s) \phi\right\| \leqslant C\|\phi\| .
$$

Proof. $L_{h}^{(k)}(t)$ is symmetric and we begin the proof by showing that $T_{h}^{(k)}(t)$ is symmetric. Since $L_{h}(t) T_{h}(t)=I$ and $T_{h}(t) L_{h}(t)=I$,

$$
T_{h}^{(k)}(t)=-\sum_{j=0}^{k-1}\left(\begin{array}{l}
k \\
j
\end{array}\right) T_{h}(t) L_{h}^{(k-j)}(t) T_{h}^{(j)}(t)
$$

and

$$
T_{h}^{(k)}(t)=-\sum_{i=1}^{k}\left(\begin{array}{c}
k \\
i
\end{array}\right) T_{h}^{(k-i)}(t) L_{h}^{(i)}(t) T_{h}(t)
$$


Therefore,

$$
\begin{aligned}
\left(T^{(k)}(t)\right)^{*} & =-\sum_{j=0}^{k-1}\left(\begin{array}{c}
k \\
j
\end{array}\right)\left(T_{h}^{(j)}(t)\right)^{*} L_{h}^{(k-j)}(t) T_{h}(t) \\
& =-\sum_{i=1}^{k}\left(\begin{array}{c}
k \\
k-i
\end{array}\right)\left(T_{h}^{(k-i)}(t)\right)^{*} L_{h}^{(i)}(t) T_{h}(t) .
\end{aligned}
$$

By induction these formulae imply that $T_{h}^{(k)}(t)$ is symmetric.

Now, if $A$ is a symmetric operator on a finite dimensional subspace of $L^{2}$ (e.g., $S_{h}$ ), then

$$
\|A\| \equiv \sup _{\phi \neq 0} \frac{\|A \phi\|}{\|\phi\|}=\sup _{\phi \neq 0} \frac{|(A \phi, \phi)|}{(\phi, \phi)} .
$$

Since the operators in (3.7) and (3.8) are symmetric, (3.7) and (3.8) are equivalent to

$$
\left|\left(T_{h}^{1 / 2}(s) L_{h}^{(k)}(t) T_{h}^{1 / 2}(s) \phi, \phi\right)\right| \leqslant C(\phi, \phi)
$$

and

$$
\left|\left(L_{h}^{1 / 2}(s) T_{h}^{(k)}(t) L_{h}^{1 / 2}(s) \phi, \phi\right)\right| \leqslant C(\phi, \phi) \quad \text { for all } \phi \in S_{h} .
$$

From (3.2),

$$
\left|\left(L_{h}^{(k)}(t) \psi, \psi\right)\right| \leqslant C\left(L_{h}(s) \psi, \psi\right) \quad \text { for all } \psi \in S_{h} .
$$

(3.12) follows with the choice $\psi=T_{h}^{1 / 2}(s) \phi$.

(3.13) is proved by induction on $k$, starting with $k=0$. From (3.12) we know that

$$
\left(T_{h}^{1 / 2}(t) L_{h}(s) T_{h}^{1 / 2}(t) \phi, \phi\right) \leqslant C(\phi, \phi) .
$$

Let $A=T_{h}^{1 / 2}(t) L_{h}(s) T_{h}^{1 / 2}(t)$. Now

$$
(A \phi, \phi) \leqslant C(\phi, \phi),
$$

where $A$ is selfadjoint and positive definite. With $\psi=A^{1 / 2} \phi(3.14)$ becomes

$$
(\psi, \psi) \leqslant C\left(A^{-1} \psi, \psi\right) \text {. }
$$

Since $A^{-1}=L_{h}^{1 / 2}(t) T_{h}(s) L_{h}^{1 / 2}(t)$,

$$
(\psi, \psi) \leqslant C\left(L_{h}^{1 / 2}(t) T_{h}(s) L_{h}^{1 / 2}(t) \psi, \psi\right) .
$$

With $\phi=T_{h}^{1 / 2}(s) L_{h}^{1 / 2}(t) \psi,(3.15)$ implies that

$$
\left(T_{h}^{1 / 2}(t) L_{h}^{1 / 2}(s) \phi, T_{h}^{1 / 2}(t) L_{h}^{1 / 2}(s) \phi\right) \leqslant C(\phi, \phi)
$$

or

$$
\left(L_{h}^{1 / 2}(s) T_{h}(t) L_{h}^{1 / 2}(s) \phi, \phi\right) \leqslant C(\phi, \phi) .
$$

Now assume (3.13) for $k \leqslant n$. From (3.9)

$$
T_{h}^{(n+1)}(t)=-\sum_{j=0}^{n}\left(\begin{array}{c}
n+1 \\
j
\end{array}\right) T_{h}(t) L_{h}^{(n+1-j)}(t) T_{h}^{(j)}(t)
$$

so that

$$
\begin{aligned}
L_{h}^{1 / 2}(s) & T_{h}^{(n+1)}(t) L_{h}^{1 / 2}(s) \\
= & -\sum_{j=0}^{n}\left(\begin{array}{c}
n+1 \\
j
\end{array}\right)\left(L_{h}^{1 / 2}(s) T_{h}(t) L_{h}^{1 / 2}(s)\right) \\
& \circ\left(T_{h}^{1 / 2}(s) L_{h}^{(n+1-j)}(t) T_{h}^{1 / 2}(s)\right)\left(L_{h}^{1 / 2}(s) T_{h}^{(j)}(t) L_{h}^{1 / 2}(s)\right) .
\end{aligned}
$$


Since the operators in parentheses are bounded (independent of $h$ ), (3.13) follows and the proof of the lemma is completed.

Note that Eq. (3.13) implies that for any $t$ and $s \in[0, \tau]$ and any integer $k \geqslant 0$

$$
\left|\left(T_{h}^{(k)}(t) \psi, \psi\right)\right| \leqslant C(k)\left(T_{h}(s) \psi, \psi\right) \text { for all } \psi \in S_{h} .
$$

(3.16) is analogous to the assumption (3.2) for $L_{h}^{(k)}(t)$ and will be used in several estimates.

We now use energy methods to bound time derivatives of $u_{h}(t)$ by the derivatives at $t=0$.

THEOREM 3.1. If $J$ is a positive integer, then the Jth time derivative $u_{h}^{(J)}$ satisfies

$$
T_{h} u_{h}^{(J+2)}+u_{h}^{(J)}+\sum_{j=0}^{J-1}\left(\begin{array}{l}
J \\
j
\end{array}\right) T_{h}^{(J-j)} u_{h}^{(j+2)}=0
$$

and for $J \geqslant 0$

$$
\begin{aligned}
& \left(T_{h} u_{h}^{(J+1)}, u_{h}^{(J+1)}\right)+\left\|u_{h}^{(J)}\right\|^{2} \\
& \quad \leqslant C\left(\sum_{j=0}^{J}\left\|u_{h}^{(j)}(0)\right\|^{2}+\sum_{j=1}^{J+1}\left(T_{h} u_{h}^{(j)}(0), u_{h}^{(j)}(0)\right)\right)
\end{aligned}
$$

Proof. (3.17) is the $J$ th time derivative of (3.4). We prove (3.18) by induction on $J$. The case $J=0$ follows by multiplying (3.4) by $u_{h}^{(1)}$ and integrating in the space variables. This procedure yields

$$
\frac{1}{2} \frac{d}{d t}\left(T_{h} u_{h}^{(1)}, u_{h}^{(1)}\right)-\frac{1}{2}\left(T_{h}^{(1)} u_{h}^{(1)}, u_{h}^{(1)}\right)+\frac{1}{2} \frac{d}{d t}\left\|u_{h}\right\|^{2}=0
$$

Integrating from 0 to $t$ gives

$$
\left(T_{h} u_{h}^{(1)}, u_{h}^{(1)}\right)+\left\|u_{h}\right\|^{2}=\left(T_{h} u_{h}^{(1)}, u_{h}^{(1)}\right)(0)+\left\|u_{h}(0)\right\|^{2}+\int_{0}^{t}\left(T_{h}^{(1)} u_{h}^{(1)}, u_{h}^{(1)}\right)(s) d s .
$$

(3.16) and Gronwall's lemma complete the proof for $J=0$.

Now, we assume (3.18) for $J \leqslant n-1$. Multiplying (3.17) by $u_{h}^{(n+1)}$ (with $J=n$ in (3.17)) and integrating in the space variables gives

$$
\begin{aligned}
\left(T_{h} u_{h}^{(n+2)},\right. & \left.u_{h}^{(n+1)}\right)+\left(u_{h}^{(n)}, u_{h}^{(n+1)}\right) \\
& +\sum_{j=0}^{n-1}\left(\begin{array}{l}
n \\
j
\end{array}\right)\left(T_{h}^{(n-j)} u_{h}^{(j+2)}, u_{h}^{(n+1)}\right)=0 .
\end{aligned}
$$

Using the identities

$$
\begin{aligned}
\left(T_{h} u_{h}^{(n+2)}, u_{h}^{(n+1)}\right)= & \frac{1}{2} \frac{d}{d t}\left(T_{h} u_{h}^{(n+1)}, u_{h}^{(n+1)}\right)-\frac{1}{2}\left(T_{h}^{(1)} u_{h}^{(n+1)}, u_{h}^{(n+1)}\right) \\
& \left(u_{h}^{(n)}, u_{h}^{(n+1)}\right)=\frac{1}{2} \frac{d}{d t}\left\|u_{h}^{(n)}\right\|^{2}
\end{aligned}
$$

and

$$
\left(T_{h}^{(n-j)} u_{h}^{(j+2)}, u_{h}^{(n+1)}\right)=\left(L_{h}^{1 / 2} T_{h}^{(n-j)} L_{h}^{1 / 2} T_{h}^{1 / 2} u_{h}^{(j+2)}, T_{h}^{1 / 2} u_{h}^{(n+1)}\right),
$$


in (3.19) and integrating from 0 to $t$ gives

$$
\begin{aligned}
\frac{1}{2}\left(T_{h} u_{h}^{(n+1)}, u_{h}^{(n+1)}\right)(t)-\frac{1}{2}\left(T_{h} u_{h}^{(n+1)}, u_{h}^{(n+1)}\right)(0) \\
+\frac{1}{2}\left\|u_{h}^{(n)}(t)\right\|^{2}-\frac{1}{2}\left\|u_{h}^{(n)}(0)\right\|^{2} \\
=\left(\frac{1}{2}-n\right) \int_{0}^{t}\left(T_{h}^{(1)} u_{h}^{(n+1)}, u_{h}^{(n+1)}\right)(s) d s \\
\quad-\sum_{j=0}^{n-2}\left(\begin{array}{l}
n \\
j
\end{array}\right) \int_{0}^{t}\left(L_{h}^{1 / 2} T_{h}^{(n-j)} L_{h}^{1 / 2} T_{h}^{1 / 2} u_{h}^{(j+2)}, T_{h}^{1 / 2} u_{h}^{(n+1)}\right) d s
\end{aligned}
$$

By Lemma 3.1 and (3.16)

$$
\left|\left(T_{h}^{(1)} u_{h}^{(n+1)}, u_{h}^{(n+1)}\right)\right| \leqslant C\left(T_{h} u_{h}^{(n+1)}, u_{h}^{(n+1)}\right)
$$

and

$$
\begin{aligned}
& \left|\left(L_{h}^{1 / 2} T_{h}^{(n-j)} L_{h}^{1 / 2} T_{h}^{1 / 2} u_{h}^{(j+2)}, T_{h}^{1 / 2} u_{h}^{(n+1)}\right)\right| \\
& \quad \leqslant C\left\|T_{h}^{1 / 2} u_{h}^{(j+2)}\right\|\left\|T_{h}^{1 / 2} u_{h}^{(n+1)}\right\| \\
& \quad \leqslant C\left(\left(T_{h} u_{h}^{(j+2)}, u_{h}^{(j+2)}\right)+\left(T_{h} u_{h}^{(n+1)}, u_{h}^{(n+1)}\right)\right) .
\end{aligned}
$$

Using these two inequalities in (3.20) yields

$$
\begin{aligned}
\left(T_{h} u_{h}^{(n+1)},\right. & \left.u_{h}^{(n+1)}\right)(t)+\left\|u_{h}^{(n)}(t)\right\|^{2} \\
\leqslant & \left(T_{h} u_{h}^{(n+1)}, u_{h}^{(n+1)}\right)(0)+\left\|u_{h}^{(n)}(0)\right\|^{2} \\
& +C \int_{0}^{t}\left(T_{h} u_{h}^{(n+1)}, u_{h}^{(n+1)}\right) d s+C \int_{0}^{t} \sum_{j=0}^{n-2}\left(T_{h} u_{h}^{(j+2)}, u_{h}^{(j+2)}\right) d s .
\end{aligned}
$$

By the induction hypothesis

$$
\sum_{j=0}^{n-2}\left(T_{h} u_{h}^{(j+2)}, u_{h}^{(j+2)}\right) \leqslant C\left(\sum_{j=0}^{n-1}\left\|u_{h}^{(j)}(0)\right\|^{2}+\sum_{j=1}^{n}\left(T_{h} u_{h}^{(j)}, u_{h}^{(j)}\right)(0)\right) .
$$

This estimate and Gronwall's lemma in (3.21) show that for any $t \in[0, \tau]$

$$
\left(T_{h} u_{h}^{(n+1)}, u_{h}^{(n+1)}\right)(t)+\left\|u_{h}^{(n)}(t)\right\|^{2} \leqslant C\left(\sum_{j=1}^{n+1}\left(T_{h} u_{h}^{(j)}, u_{h}^{(j)}\right)(0)+\sum_{j=0}^{n}\left\|u_{h}^{(j)}(0)\right\|^{2}\right) \text {. }
$$

This proves the theorem.

The next theorem is a bound for time derivatives of $u(t)-u_{h}(t)$ in $L^{2}$. Let $e(t)=u(t)-u_{h}(t)$ and, for positive integer $J, e^{(J)}(t)=u^{(J)}(t)-u_{h}^{(J)}(t)$. Subtracting $T_{h}\left(u_{h}\right)_{t t}+u_{h}=0$ from $T u_{t t}+u=0$ gives the following error equation.

$$
T_{h} e^{(2)}+e=\left(T_{h}-T\right) u_{t t} \equiv \rho .
$$

THEOREM 3.2. Let $J$ be a positive integer. The solution e of (3.22) satisfies

$$
T_{h} e^{(J+2)}+e^{(J)}+\sum_{j=0}^{J-1}\left(\begin{array}{l}
J \\
j
\end{array}\right) T_{h}^{(J-j)} e^{(j+2)}=\rho^{(J)}
$$


and for $t \in[0, \tau]$ and $J \geqslant 0$

$$
\begin{aligned}
& \left(T_{h} e^{(J+1)}, e^{(J+1)}\right)(t)+\left\|e^{(J)}(t)\right\|^{2} \\
& \leqslant C\left(\sum_{j=1}^{J+1}\left(T_{h} e^{(j)}, e^{(j)}\right)(0)+\sum_{j=0}^{J}\left\|e^{(j)}(0)\right\|^{2}\right. \\
& \left.\quad+\sup _{0 \leqslant s^{\prime} \leqslant t}\left(\sum_{j=0}^{J}\left\|\rho^{(j)}\left(s^{\prime}\right)\right\|^{2}+\sum_{j=1}^{J+1} \int_{0}^{s^{\prime}}\left\|\rho^{(j)}\right\|^{2} d \sigma\right)\right) .
\end{aligned}
$$

Furthermore, for $2 \leqslant s \leqslant r$,

$$
\begin{aligned}
&\left(T_{h} e^{(J+1)}, e^{(J+1)}\right)(t)+\left\|e^{(J)}(t)\right\|^{2} \\
& \leqslant C\left(\sum_{j=1}^{J+1}\left(T_{h} e^{(j)}, e^{(j)}\right)(0)+\sum_{j=0}^{J}\left\|e^{(j)}(0)\right\|^{2}\right. \\
&\left.+h^{2 s}\left(\left\|u^{0}\right\|_{J+s+1}+\left\|u_{t}^{0}\right\|_{J+s}\right)^{2}\right) .
\end{aligned}
$$

Proof. The proof is similar to the proof of Theorem 3.1. Here $e$ replaces $u_{h}$ in Theorem 3.1 and $\rho$ replaces 0 on the right-hand side. (3.23) follows by differentiating (3.22) $J$ times. With $T_{h}^{(j)}=T_{h}^{(j)} P$ and $T_{h}^{1 / 2}=T_{h}^{1 / 2} P$, where $P$ is the $L^{2}$ orthogonal projection onto $S_{h}$, the same estimates for $T_{h}^{(j)}$ that were used in the proof of Theorem 3.1 prove that

$$
\begin{aligned}
&\left(T_{h} e^{(J+1)}, e^{(J+1)}\right)(t)+\left\|e^{(J)}(t)\right\|^{2} \\
& \leqslant\left(T_{h} e^{(J+1)}, e^{(J+1)}\right)(0)+\left\|e^{(J)}(0)\right\|^{2} \\
&+C\left(\int_{0}^{t}\left(T_{h} e^{(J+1)}, e^{(J+1)}\right) d s+\int_{0}^{t} \sum_{j=0}^{J-2}\left(T_{h} e^{(j+2)}, e^{(j+2)}\right) d s\right) \\
&+2 \int_{0}^{t}\left(\rho^{(J)}, e^{(J+1)}\right) d s .
\end{aligned}
$$

Since

$$
\left(\rho^{(J)}, e^{(J+1)}\right)=\frac{d}{d t}\left(\rho^{(J)}, e^{(J)}\right)-\left(\rho^{(J+1)}, e^{(J)}\right)
$$

it follows that

$$
\int_{0}^{t}\left(\rho^{(J)}, e^{(J+1)}\right) d s=\left(\rho^{(J)}, e^{(J)}\right)(t)-\left(\rho^{(J)}, e^{(J)}\right)(0)-\int_{0}^{t}\left(\rho^{(J+1)}, e^{(J)}\right) d s
$$

and

$$
\begin{aligned}
2 \int_{0}^{t}\left(\rho^{(J)}, e^{(J+1)}\right) d s \leqslant & 2\left\|\rho^{(J)}(t)\right\|^{2}+\frac{1}{2}\left\|e^{(J)}(t)\right\|^{2} \\
& +\left\|\rho^{(J)}(0)\right\|^{2}+\left\|e^{(J)}(0)\right\|^{2}+\int_{0}^{t}\left\|\rho^{(J+1)}\right\|^{2} d s \\
& +\int_{0}^{t}\left\|e^{(J)}\right\|^{2} d s .
\end{aligned}
$$


(3.24) is proved by induction. For $J=0$ (3.26) and (3.27) give

$$
\begin{aligned}
\left(T_{h} e^{(1)}, e^{(1)}\right)+\|e\|^{2} \leqslant & \left(T_{h} e^{(1)}, e^{(1)}\right)(0)+\|e(0)\|^{2} \\
& +C \int_{0}^{t}\left(T_{h} e^{(1)}, e^{(1)}\right) d s+\int_{0}^{t}\|e\|^{2} d s \\
& +2\|\rho(t)\|^{2}+\|\rho(0)\|^{2}+\frac{1}{2}\|e(t)\|^{2}+\|e(0)\|^{2}+\int_{0}^{t}\left\|\rho^{(1)}\right\|^{2} d s .
\end{aligned}
$$

Subtracting $\frac{1}{2}\|e(t)\|^{2}$ from both sides and Gronwall's lemma give (3.24) with $J=0$.

Now we assume (3.24) for $J \leqslant n-1$ and use (3.27) in (3.26) (with $J=n$ in (3.26) and (3.27)) to obtain

$$
\begin{aligned}
&\left(T_{h} e^{(n+1)}, e^{(n+1)}\right)(t)+\left\|e^{(n)}(t)\right\|^{2} \\
& \leqslant\left(T_{h} e^{(n+1)}, e^{(n+1)}\right)(0)+\left\|e^{(n)}(0)\right\|^{2} \\
&+C\left(\sum_{j=1}^{n}\left(T_{h} e^{(j)}, e^{(j)}\right)(0)+\sum_{j=0}^{n-1}\left\|e^{(j)}(0)\right\|^{2}\right. \\
&\left.+\sup _{0 \leqslant s^{\prime} \leqslant t}\left(\sum_{j=0}^{n-1}\left\|\rho^{(j)}\left(s^{\prime}\right)\right\|^{2}+\sum_{j=1}^{n} \int_{0}^{s^{\prime}}\left\|\rho^{(j)}\right\|^{2} d \sigma\right)\right) \\
&+2\left\|\rho^{(n)}(t)\right\|^{2}+\left\|\rho^{(n)}(0)\right\|^{2}+\frac{1}{2}\left\|e^{(n)}(t)\right\|^{2}+\left\|e^{(n)}(0)\right\|^{2} \\
&+\int_{0}^{t}\left\|\rho^{(n+1)}\right\|^{2} d s+C \int_{0}^{t}\left(T_{h} e^{(n+1)}, e^{(n+1)}\right) d s+\int_{0}^{t}\left\|e^{(n)}\right\|^{2} d s .
\end{aligned}
$$

Subtracting $\frac{1}{2}\left\|e^{(n)}(t)\right\|^{2}$ from both sides of (3.28) and using Gronwall's lemma gives

$$
\begin{aligned}
& \left(T_{h} e^{(n+1)}, e^{(n+1)}\right)(t)+\left\|e^{(n)}(t)\right\|^{2} \\
& \leqslant C\left(\sum_{j=1}^{n+1}\left(T_{h} e^{(j)}, e^{(j)}\right)(0)+\sum_{j=0}^{n}\left\|e^{(j)}(0)\right\|^{2}\right. \\
& \left.\quad+\sup _{0 \leqslant s^{\prime} \leqslant t}\left(\sum_{j=0}^{n}\left\|\rho^{(j)}\left(s^{\prime}\right)\right\|^{2}+\sum_{j=1}^{n+1} \int_{0}^{s^{\prime}}\left\|\rho^{(j)}\right\|^{2} d \sigma\right)\right)
\end{aligned}
$$

and this is (3.24) with $J=n$.

To complete the proof of the theorem we show that (3.25) follows from (3.24). This requires estimating the terms

$$
\sum_{j=0}^{J}\left\|\rho^{(j)}\left(s^{\prime}\right)\right\|^{2}+\sum_{j=1}^{J+1} \int_{0}^{s^{\prime}}\left\|\rho^{(j)}(\sigma)\right\|^{2} d \sigma .
$$

When the terms $\rho^{(j)}$ appearing in (3.29) are estimated using Leibniz's rule and the triangle inequality, the resulting terms which require the most regularity on $u$ are

$$
\left\|\left(T_{h}-T\right) u^{(J+2)}\left(s^{\prime}\right)\right\|^{2} \text { and } \int_{0}^{s^{\prime}}\left\|\left(T_{h}-T\right) u^{(J+3)}(\sigma)\right\|^{2} d \sigma .
$$

From (3.1)

$$
\left\|\left(T_{h}-T\right) u^{(J+2)}\left(s^{\prime}\right)\right\| \leqslant C h^{s}\left\|u^{(J+2)}\left(s^{\prime}\right)\right\|_{s-2}
$$


and

$$
\int_{0}^{s^{\prime}}\left\|\left(T_{h}-T\right) u^{(J+3)}(\sigma)\right\|^{2} d \sigma \leqslant C h^{2 s} \int_{0}^{s^{\prime}}\left\|u^{(J+3)}(\sigma)\right\|_{s-2}^{2} d \sigma
$$

and from (2.15) it follows that for $0 \leqslant s^{\prime} \leqslant t \leqslant \tau$,

$$
\begin{gathered}
\left\|u^{(J+2)}\left(s^{\prime}\right)\right\|_{s-2} \leqslant C\left(\left\|u^{0}\right\|_{J+s+1}+\left\|u_{t}^{0}\right\|_{J+s}\right), \\
\int_{0}^{s^{\prime}}\left\|u^{(J+3)}(\sigma)\right\|_{s-2}^{2} d \sigma \leqslant C\left(\left\|u^{0}\right\|_{J+s+1}+\left\|u_{t}^{0}\right\|_{J+s}\right)^{2} .
\end{gathered}
$$

All other terms which are produced by using Leibniz's rule in (3.29) are bounded by $C h^{2 s}\left(\left\|u^{0}\right\|_{J+s+1}+\left\|u_{t}^{0}\right\|_{J+s}\right)^{2}$ and therefore

$$
\sup _{0 \leqslant s^{\prime} \leqslant t}\left(\sum_{j=0}^{J}\left\|\rho^{(j)}\left(s^{\prime}\right)\right\|^{2}+\sum_{j=1}^{J+1} \int_{0}^{s^{\prime}}\left\|\rho^{(j)}\right\|^{2} d \sigma\right) \leqslant C h^{2 s}\left(\left\|u^{0}\right\|_{J+s+1}+\left\|u_{t}^{0}\right\|_{J+s}\right)^{2} .
$$

This proves (3.25) and completes the proof of the theorem.

Special initial data will be required so that the terms

$$
\sum_{j=1}^{J+1}\left(T_{h} e^{(j)}, e^{(j)}\right)(0)+\sum_{j=0}^{J}\left\|e^{(j)}(0)\right\|^{2}
$$

can be bounded by $h^{2 s}$. The initial data will be chosen so that fully discrete error estimates can be proved also (see Section 4). The special initial data is the subject of Section 5 and is motivated by work in Sammon [20] and [21].

4. Single Step Fully Discrete Approximations. In this section discretization of (3.4) in the time variable is analyzed. (3.4) is a system of ordinary differential equations and a class of single step methods is applied to these ordinary differential equations. For the single step methods the interval $[0, \tau]$ is divided into $N$ equal subintervals of length $k$ and $t_{n} \equiv n k$ for $n=0,1, \ldots, N$. For any smooth function $y(t)$ the methods (which are called Obrechkoff methods (see Lambert [16])) are defined by the formula

$$
y_{n+1}-y_{n}=k\left(-q_{1} y_{n+1}^{\prime}+p_{1} y_{n}^{\prime}\right)+k^{2}\left(-q_{2} y_{n+1}^{\prime \prime}+p_{2} y_{n}^{\prime \prime}\right),
$$

where $y_{m}, y_{m}^{\prime}$, and $y_{m}^{\prime \prime}$, for $m=0,1, \ldots, N$, approximate $y\left(t_{m}\right), d y\left(t_{m}\right) / d t$ and $d^{2} y\left(t_{m}\right) / d t^{2}$, respectively, and $p_{i}, q_{i}$, for $i=1,2$, are given constants. The function $\mathscr{A}[y]$ defined by

$$
\begin{aligned}
\mathscr{A}[y]= & y(t+k)-y(t)+k\left(q_{1} y^{\prime}(t+k)-p_{1} y^{\prime}(t)\right) \\
& +k^{2}\left(q_{2} y^{\prime \prime}(t+k)-p_{2} y^{\prime \prime}(t)\right)
\end{aligned}
$$

is associated with (4.1). $\mathscr{A}[y]$ is the truncation error of the single step methods and will be used to define the order of a given method.

Definition 4.1. A method given by (4.1) is of order $\nu>0$, if $\mathscr{A}\left[t^{j}\right]=0$ for $j=0,1, \ldots, \nu$, and $\mathscr{A}\left[t^{\nu+1}\right] \neq 0$.

Definition 4.2. The stability region $R$ associated with a method given by (4.1) is defined as $R=\{k \lambda$ : where $\lambda$ is any complex number and $k$ any positive number such that when the method is applied to $y^{\prime}=\lambda y$ with $y\left(x_{0}\right)=y_{0}$ given and with constant step size $k$, the sequence $\left\{y_{n}\right\}_{n=1}^{N}$ satisfies $\left.\left|y_{n}\right| \leqslant\left|y_{0}\right|\right\}$. 
For the methods given by (4.1), $1 \leqslant \nu \leqslant 4$. We will assume that $q_{2}=p_{2}=0$ in (4.1) when $\nu=1$. Also, in this work we will assume that the stability region $R$ contains the imaginary axis, i.e., the methods are unconditionally stable. The latter assumption implies that the rational function

$$
r(x)=\left(1+p_{1} x+p_{2} x^{2}\right) /\left(1+q_{1} x+q_{2} x^{2}\right)
$$

satisfies $|r(\mu)| \leqslant 1$ for any purely imaginary number $\mu$. This implies that $1+q_{1} x+$ $q_{2} x^{2}$ has no zeros on the imaginary axis.

Examples of methods satisfying the above assumptions are given in the following table.

Corresponding Rational

Approximation to Exponential

Backward Euler

$\begin{array}{ccccc}\nu & q_{1} & q_{2} & p_{1} & p_{2} \\ 1 & -1 & 0 & 0 & 0 \\ 2 & -\frac{1}{2} & 0 & \frac{1}{2} & 0\end{array}$

Crank-Nicolson

$3-2 \lambda \quad \lambda^{2} \quad 1-2 \lambda \quad \lambda^{2}-2 \lambda+\frac{1}{2}$

Calahan*

Padé

Padé

$\begin{array}{lll}2 & -1 & \frac{1}{2}\end{array}$

Padé

$3-\frac{2}{3} \quad \frac{1}{6}$

$4-\frac{1}{2} \quad \frac{1}{12}$

0

$* \lambda=\frac{1}{2}(1+1 / \sqrt{3})$.

The definitions given above for order and stability are used in the theory of numerical methods for ordinary differential equations (see Lambert [16]). In the case of linear second order hyperbolic partial differential equations with time-independent coefficients, these definitions reduce to the ones used by Baker and Bramble [2], where the single step methods (4.1) define rational approximations to the exponential.

The following lemma is an analysis of the truncation error (4.2).

LEMMA 4.1. If the single step method (4.1) has order $\nu$ and $y(t)$ is a function with $\nu+1$ time derivatives then

$$
\begin{aligned}
\mathscr{A}[y]= & \int_{t}^{t+k} \frac{(t+k-s)^{\nu}}{\nu !} y^{(\nu+1)}(s) d s \\
& +q_{1} k \int_{t}^{t+k} \frac{(t+k-s)^{\nu-1}}{(\nu-1) !} y^{(\nu+1)}(s) d s \\
& +q_{2} k^{2} \int_{t}^{t+k} \frac{(t+k-s)^{\nu-2}}{(\nu-2) !} y^{(\nu+1)}(s) d s .
\end{aligned}
$$

Proof. (4.3) follows from (4.2) by expanding the terms which contain $t+k$ in a Taylor series about $t$ and using the fact that $\mathscr{A}\left[t^{j}\right]=0$ for $j=0, \ldots, \nu$. If $\nu=1$, the last term in (4.3) does not appear.

The two polynomials $p(x)=1+p_{1} x+p_{2} x^{2}$ and $q(x)=1+q_{1} x+q_{2} x^{2}$ are associated with the single step methods. Note that $p(x) / q(x)$ is the rational approximation to the exponential which was analyzed for linear parabolic partial differential equations with time-independent coefficients in Baker, Bramble, and 
Thomée [3] and for second order linear hyperbolic partial differential equations with time-independent coefficients in Baker and Bramble [2]. We denote the degree of $q(x)$ and the degree of $p(x)$ by $\operatorname{deg} q$ and $\operatorname{deg} p$, respectively.

In this section in addition to properties (i), (ii), and (iii) (see Section 3 ) of the approximate solution operator $T_{h}$, if $\operatorname{deg} q=2$, we will assume the following inverse property on $S_{h}$ :

(iv) $\left(L_{h} \phi, \phi\right)^{1 / 2} \leqslant C h^{-1}\|\phi\|$.

We note that (iv) implies that

$$
\begin{gathered}
\left\|L_{h} \phi\right\| \leqslant C h^{-2}\|\phi\|, \\
\left\|L_{h}^{(l)}(s) T_{h}(t) \phi\right\| \leqslant C\|\phi\|,
\end{gathered}
$$

and

$$
\left\|T_{h}(t) L_{h}^{(l)}(s) \phi\right\| \leqslant C\|\phi\|,
$$

for integer $l \geqslant 0$, for $s, t \in[0, \tau]$, and for all $\phi \in S_{h}$. See Sammon [20] and [21] and Section 7 for more details on these estimates.

(3.4) can be written as a first order system of ordinary differential equations so that the single step methods (4.1) can be used to discretize the ordinary differential equations.

With

$$
U_{h} \equiv\left(\begin{array}{c}
u_{h} \\
\left(u_{h}\right)_{t}
\end{array}\right) \text { and } \quad \mathscr{L}_{h} \equiv\left(\begin{array}{cc}
0 & I \\
-L_{h} & 0
\end{array}\right)
$$

(3.4) becomes

$$
\left(U_{h}\right)_{t}=\mathscr{L}_{h} U_{h}, \quad 0 \leqslant t \leqslant \tau, \quad U_{h}(0)=\left(\begin{array}{c}
v^{0} \\
v_{t}^{0}
\end{array}\right) .
$$

We now think of $y_{k}$ in (4.1) as approximating $U_{h}\left(t_{k}\right)$ for $k=0, \ldots, N$, and $y_{k}^{\prime}$ approximating $U_{h}^{(1)}\left(t_{k}\right)$, etc. (4.1) requires the second time derivative of $U_{h}$ which is obtained by differentiating (4.7),

$$
\left(U_{h}\right)_{t}=\mathscr{L}_{h} U_{h}, \quad\left(U_{h}\right)_{t t}=\left(\mathscr{L}_{h}^{2}+\mathscr{L}_{h}^{(1)}\right) U_{h} .
$$

The following notation will be used so that the equation which is derived from formally substituting (4.8) into (4.1) can be written in a convenient form.

For $j=0, \ldots, N$,

$$
\begin{aligned}
& Q_{j} \equiv I+q_{1} k \mathscr{L}_{h}\left(t_{j}\right)+q_{2} k^{2} \mathscr{L}_{h}^{2}\left(t_{j}\right), \quad \tilde{Q}_{j} \equiv Q_{j}+q_{2} k^{2} \mathscr{L}_{h}^{(1)}\left(t_{j}\right), \\
& P_{j} \equiv I+p_{1} k \mathscr{L}_{h}\left(t_{j}\right)+p_{2} k^{2} \mathscr{L}_{h}^{2}\left(t_{j}\right) \text { and } \quad \tilde{P}_{j} \equiv P_{j}+p_{2} k^{2} \mathscr{L}_{h}^{(1)}\left(t_{j}\right) .
\end{aligned}
$$

For integer $i \geqslant 0$,

$$
\begin{aligned}
& L_{j}^{(i)} \equiv L_{h}^{(i)}\left(t_{j}\right), \quad T_{j}^{(i)} \equiv T_{h}^{(i)}\left(t_{j}\right), \\
& \mathscr{L}_{j}^{(i)} \equiv \mathscr{L}_{h}^{(i)}\left(t_{j}\right) \quad \text { and } \quad \mathscr{T}_{j}^{(i)} \equiv\left(\mathscr{L}_{h}^{-1}\right)^{(i)}\left(t_{j}\right) \text {. }
\end{aligned}
$$

Note that $\mathscr{L}_{h}$ is a linear operator from $S_{h} \times S_{h}$ to $S_{h} \times S_{h}$ and

$$
\mathscr{T}_{h} \equiv\left(\mathscr{L}_{h}\right)^{-1}=\left(\begin{array}{cc}
0 & -T_{h} \\
I & 0
\end{array}\right) \text {. }
$$


The fully discrete approximation to (1.1) is defined as $\left\{W^{n}\right\}_{n=0}^{N} \subset S_{h} \times S_{h}$ which satisfies

$$
\tilde{Q}_{n+1} W^{n+1}=\tilde{P}_{n} W^{n},
$$

where $W^{0}$ is given in $S_{h} \times S_{h}$. We will show that $\tilde{Q}_{n+1}$ is invertible, if $k$ is small enough, so that $W^{n}$ for $n \geqslant 1$ is well-defined. We will also derive error estimates for

$$
W^{n}-\left(\begin{array}{c}
u_{h}\left(t_{h}\right) \\
\left(u_{h}\right)_{t}\left(t_{n}\right)
\end{array}\right) .
$$

These error estimates will be used together with the semidiscrete error estimates and the triangle inequality to estimate $\left\|u\left(t_{n}\right)-W_{1}^{n}\right\|$, where $W_{1}^{n} \in S_{h}$ is the first component of $W^{n}$. This entire analysis will be done in a special inner product on $S_{h} \times S_{h}$ which will be denoted by

$$
((\Phi, \psi))_{n}=\left(\phi_{1}, \bar{\psi}_{1}\right)+\left(T_{h}\left(t_{n}\right) \phi_{2}, \bar{\psi}_{2}\right)
$$

where

$$
\Phi \equiv\left(\begin{array}{l}
\phi_{1} \\
\phi_{2}
\end{array}\right) \text { and } \psi \equiv\left(\begin{array}{l}
\psi_{1} \\
\psi_{2}
\end{array}\right)
$$

can be complex-valued functions and $\bar{\psi}_{1}$ and $\bar{\psi}_{2}$ denote the complex conjugate of $\psi_{1}$ and $\psi_{2}$, respectively. The corresponding norm is denoted by

$$
\|\Phi\|_{n}=((\Phi, \Phi))_{n}^{1 / 2} \text { for } n=0,1, \ldots, N .
$$

Note that from (3.16) it follows that the norms $\||\cdot|\|_{m}$ and $\|\mid \cdot\| \|_{n}$ are equivalent for any integers $m$ and $n$ between 0 and $N$.

The following lemma proves that $Q_{n}$ is invertible.

LEMMA 4.2. For any nonzero complex number $\alpha$ and for all $\Phi \in S_{h} \times S_{h}$

$$
\frac{|\operatorname{Re} \alpha|}{|\alpha|}\|\Phi\|_{n} \leqslant\left\|\left(I+\alpha k \mathscr{L}_{n}\right) \Phi\right\|_{n}
$$

and

$$
|\operatorname{Re} \alpha|\left\|k \mathscr{L}_{n} \Phi\left|\left\|_{n} \leqslant\right\|\right|\left(I+\alpha k \mathscr{L}_{n}\right) \Phi \mid\right\|_{n},
$$

where $\operatorname{Re} \alpha$ denotes the real part of $\alpha$. If $q(x)=1+\alpha x$, then

$$
\frac{|\operatorname{Re} \alpha|}{|\alpha|}\|\Phi \mid\|_{n} \leqslant\left\|Q_{n} \Phi\right\|_{n}
$$

and

$$
|\operatorname{Re} \alpha|\left\|k \mathscr{L}_{n} \Phi\right\||\leqslant| \mid Q_{n} \Phi \|_{n},
$$

and if $q(x)=\left(1+\alpha_{1} x\right)\left(1+\alpha_{2} x\right)$, then

$$
\begin{gathered}
\frac{\left|\operatorname{Re} \alpha_{1}\right|\left|\operatorname{Re} \alpha_{2}\right|}{\left|\alpha_{1}\right|\left|\alpha_{2}\right|}\|\Phi\| \leqslant\left\|Q_{n} \Phi\right\|_{n}, \\
\frac{\left|\operatorname{Re} \alpha_{1}\right|}{\left|\alpha_{1}\right|}\left|\operatorname{Re} \alpha_{2}\right|\left\|k \mathscr{L}_{n} \Phi\right\|_{n} \leqslant\left\|Q_{n} \Phi\right\|_{n},
\end{gathered}
$$

and

$$
\left|\operatorname{Re} \alpha_{1}\right|\left|\operatorname{Re} \alpha_{2}\right|||\left|k^{2} \mathscr{L}_{n}^{2} \Phi\right||\leqslant|\left\|Q_{n} \Phi \mid\right\|_{n} .
$$


Proof. (4.14)-(4.17) and (4.18) are applications of (4.12) and (4.13). It suffices to prove (4.12) and (4.13).

Let $\left\{\phi_{j}\right\}_{j=1}^{m}$ and $\left\{\lambda_{j}\right\}_{j=1}^{m}$ be the orthonormal eigenfunctions and eigenvalues of $L_{n} \cdot \mathscr{L}_{n}=\left(\begin{array}{cc}0 & l \\ -L_{n} & 0\end{array}\right)$ has orthonormal eigenfunctions

$$
\Phi_{ \pm j}=\frac{1}{\sqrt{2}}\left(\begin{array}{c}
\phi_{j} \\
\pm i \sqrt{\lambda_{j}} \phi_{j}
\end{array}\right)
$$

(which are a complete set) and eigenvalues $\pm i \sqrt{\lambda}_{j}$, for $j=1, \ldots, m$. With $\Phi=$ $\sum_{j=-m}^{m} C_{j} \Phi_{j}$,

$$
\left(I+\alpha k \mathscr{L}_{n}\right) \Phi=\sum_{j=-m}^{m} C_{j}(1+\alpha k(\operatorname{sgn} j) i \sqrt{\lambda}|j|) \Phi_{j},
$$

where $\operatorname{sgn} j$ is the sign of $j$. We have

$$
\left\|\left(I+\alpha k \mathscr{L}_{n}\right) \Phi\right\|_{n}^{2}=\sum_{j=-m}^{m}\left|C_{j}\right|^{2}|1+\alpha k(\operatorname{sgn} j) i \sqrt{\lambda}| j||^{2}
$$

and

$$
\left\|\left(I+\alpha k \mathscr{L}_{n}\right) \Phi\right\|_{n}^{2}=\sum_{j=-m}^{m}|k i \sqrt{\lambda}| j||^{2}\left|C_{j}\right|^{2} \frac{|1+\alpha k(\operatorname{sgn} j) i \sqrt{\lambda}| j||^{2}}{|k i \sqrt{\lambda}| j||^{2}} .
$$

Let $x=k \sqrt{\lambda}_{|j|}$ sgn $j$. Since the functions $f_{1}(x)=|1+\alpha i x|^{2}$ and $f_{2}(x)=$ $|1+\alpha i x|^{2} / x^{2}$ satisfy $f_{1}(x) \geqslant|\operatorname{Re} \alpha|^{2} /|\alpha|^{2}$ and $f_{2}(x) \geqslant|\operatorname{Re} \alpha|^{2}$ for all real $x$, it follows from (4.19) and (4.20) that

$$
\left\|\left(I+\alpha k \mathscr{L}_{n}\right) \Phi\right\|_{n}^{2} \geqslant \sum_{j=-m}^{m}\left|C_{j}\right|^{2} \frac{|\operatorname{Re} \alpha|^{2}}{|\alpha|^{2}}
$$

and

$$
\left\|\left(I+\alpha k \mathscr{L}_{n}\right) \Phi\right\|_{n}^{2} \geqslant \sum_{j=-m}^{m}|k i \sqrt{\lambda}| j||^{2}\left|C_{j}\right|^{2}|\operatorname{Re} \alpha|^{2} .
$$

These estimates give (4.12) and (4.13) and complete the proof of the lemma.

The next lemma proves that if $k$ is small enough then $\tilde{Q}_{n}$ is invertible (when $q(x)$ is quadratic).

LEMMA 4.3. If $k$ is sufficiently small and $q_{2} \neq 0$, then for all $\Phi \in S_{h} \times S_{h}$,

$$
C_{1}\left\|Q_{n} \Phi\right\|_{n} \leqslant\left\|\tilde{Q}_{n} \Phi\right\|_{n} \leqslant C_{2}\left\|Q_{n} \Phi\right\|_{n},
$$

where $C_{1}$ and $C_{2}$ are independent of the step lengths $h$ and $k$.

Proof.

$$
\left\|\left(\tilde{Q}_{n}-Q_{n}\right) \Phi\right\|_{n}=\|\| q_{2} k^{2} \mathscr{L}_{n}^{(1)} \Phi \|_{n}
$$

It is easy to see that

$$
k \mathscr{L}_{n}^{(1)} \mathscr{T}_{n} \psi=k\left(\begin{array}{c}
0 \\
L_{n}^{(1)} T_{n} \psi_{2}
\end{array}\right)
$$

and

$$
\left\|\mathscr{L}_{n}^{(1)} \mathscr{T}_{n} \psi\right\|_{n}^{2}=\left\|T_{n}^{1 / 2} L_{n}^{(1)} T_{n} \psi_{2}\right\|^{2}=\left\|T_{n}^{1 / 2} L_{n}^{(1)} T_{n}^{1 / 2} T_{n}^{1 / 2} \psi\right\|^{2}
$$


From this identity and Lemma 3.1,

$$
\left\|\mathscr{L}_{n}^{(1)} \mathscr{T}_{n} \psi\right\|_{n}^{2} \leqslant C\left(T_{n}^{1 / 2} \psi_{2}, T_{n}^{1 / 2}, \psi_{2}\right)=C\left(T_{n} \psi_{2}, \psi_{2}\right)
$$

Therefore, $k\|\| \mathscr{L}_{n}^{(1)} \mathscr{T}_{n} \psi\left|\left\|_{n} \leqslant C k\right\|\|\psi \mid\|_{n}\right.$. Substituting $\Phi=\mathscr{T}_{n} \psi$ gives $k\|\| \mathscr{L}_{n}^{(1)} \Phi \|_{n} \leqslant$ $C k\left|\left\|\mathscr{L}_{n} \Phi \mid\right\|_{n}\right.$. From this estimate, (4.22) and Lemma 4.2 (from the stability assumption $q(x)$ has no roots on the imaginary axis) we have

$$
\left\|\left(\tilde{Q}_{n}-Q_{n}\right) \Phi\right\|_{n} \leqslant C k\left\|Q_{n} \Phi\right\|_{n} .
$$

The triangle inequality gives

$$
(1-C k)\left\|Q_{n} \Phi\right\|_{n} \leqslant\left\|\tilde{Q}_{n} \Phi\right\|_{n} \leqslant(1+C k)\left\|Q_{n} \Phi\right\|_{n} .
$$

This estimate gives (4.21) for sufficiently small $k$ and completes the proof.

The fully discrete scheme defined by (4.9) is well-defined since $Q_{n+1}$ and $\tilde{Q}_{n+1}$ are invertible. We now analyze the error $\left\|\mid U_{h}\left(t_{n}\right)-W^{n}\right\|_{n}$ by studying the following error equation. Define $E^{n}=U_{h}\left(t_{n}\right)-W^{n}$ for $n=0,1, \ldots, N$. By manipulation of (4.9) we have

$$
\begin{aligned}
Q_{n+1} E^{n+1}= & P_{n+1} E^{n}+\left(P_{n}-P_{n+1}\right) E^{n} \\
& +\left(\tilde{P}_{n}-P_{n}\right) E^{n}+\left(Q_{n+1}-\tilde{Q}_{n+1}\right) E^{n+1} \\
& +\tilde{Q}_{n+1} U_{h}\left(t_{n+1}\right)-\tilde{P}_{n} U_{h}\left(t_{n}\right) .
\end{aligned}
$$

The next four lemmas bound terms in (4.23).

LEMMA 4.4.

$$
\left\|P_{n+1} E^{n}\right\|\left\|_{n+1} \leqslant(1+C k)\right\| Q_{n} E^{n} \|_{n}
$$

Proof. $P_{n+1} E^{n}=Q_{n+1}^{-1} P_{n+1} Q_{n+1} E^{n}$ and, with $\psi=Q_{n+1} E^{n}=\sum_{j=-m}^{m} C_{j} \Phi_{j}$,

$$
\left\|\left.Q_{n+1}^{-1} P_{n+1} \psi\left|\|_{n+1}^{2}=\sum_{j=-m}^{m}\right| C_{j}\right|^{2}\left|\frac{p((\operatorname{sgn} j) i \sqrt{\lambda}|j|)}{q((\operatorname{sgn} j) i \sqrt{\lambda}|j|)}\right|^{2},\right.
$$

where $\left\{\Phi_{j}\right\}_{j=-m}^{m}$ are the eigenfunctions and $\left\{(\operatorname{sgn} j) i \sqrt{\lambda}_{|j|}\right\}_{j=-m}^{m}$ are the eigenvalues of $\mathscr{L}_{n+1}$, which were introduced in the proof of Lemma 4.2. From the stability assumption, it follows that if $\operatorname{Re} \mu=0$, then $|p(\mu) / q(\mu)| \leqslant 1$. Hence, the stability assumption and (4.25) imply that $\left\|Q_{n+1}^{-1} P_{n+1} \psi\right\|_{n+1}^{2} \leqslant\|\psi\| \|_{n+1}^{2}$. Substituting $\psi=$ $Q_{n+1} E^{n}$ gives

$$
\left\|P_{n+1} E^{n}\right\|_{n+1}^{2} \leqslant\left\|Q_{n+1} E^{n}\right\|_{n+1}^{2} .
$$

(4.24) follows from (4.26) and the following lemma.

LeMma 4.5. For any $\Phi \in S_{h} \times S_{h}$,

$$
\begin{gathered}
\|\Phi\|_{n+1} \leqslant(1+C k)\|\Phi\|_{n}, \\
\left\|\left(P_{n}-P_{n+1}\right) E^{n}\right\|_{n+1} \leqslant C k\left\|Q_{n} E^{n}\right\|_{n}
\end{gathered}
$$

and

$$
\left\|\left(Q_{n}-Q_{n+1}\right) E^{n}\right\|_{n+1} \leqslant C k\left\|Q_{n} E^{n}\right\|_{n} .
$$


Proof. To prove (4.27) note that since

$$
\begin{aligned}
\|\Phi\|_{n+1}^{2}=\left(\left(\phi_{1}, \phi_{1}\right)+\right. & \left.\left(T_{n+1} \phi, \phi_{2}\right)\right) \quad \text { and }\|\Phi\|_{n}^{2}=\left(\left(\phi_{1}, \phi_{1}\right)+\left(T_{n} \phi_{2}, \phi_{2}\right)\right), \\
\|\Phi\|_{n+1}^{2}-\|\Phi\|_{n}^{2} & =\left(\left(T_{n+1}-T_{n}\right) \phi_{2}, \phi_{2}\right) \\
& =\int_{t_{n}}^{t_{n+1}}\left(L_{n}^{1 / 2} T_{h}^{(1)}(s) L_{n}^{1 / 2} T_{n}^{1 / 2} \phi_{2}, T_{n}^{1 / 2} \phi_{2}\right) d s
\end{aligned}
$$

So by Lemma 3.1,

$$
\|\Phi\|_{n+1}^{2} \leqslant\|\Phi\|_{n}^{2}+C k\left(T_{n} \phi_{2}, \phi_{2}\right) \leqslant(1+C k)\|\Phi\|_{n}^{2}
$$

and

$$
\|\Phi\|_{n+1} \leqslant(1+C k)^{1 / 2}\|\Phi\|_{n} \leqslant(1+C k / 2)\|\Phi\|_{n} .
$$

This proves (4.27). To prove (4.28) and (4.29) we first show that

$$
\left\|k\left(\mathscr{L}_{n+1}-\mathscr{L}_{n}\right) \Phi\right\|_{n+1} \leqslant C k\left\|Q_{n} \Phi\right\|_{n}
$$

and

$$
\left\|k^{2}\left(\mathscr{L}_{n+1}^{2}-\mathscr{L}_{n}^{2}\right) \Phi\right\|_{n+1} \leqslant C k\left\|Q_{n} \Phi\right\|_{n}
$$

Note that

$\mathscr{L}_{n+1}-\mathscr{L}_{n}=\int_{t_{n}}^{t_{n+1}} \mathscr{L}_{n}^{(1)}(s) d s \quad$ and $\quad \mathscr{L}_{n+1}^{2}-\mathscr{L}_{n}^{2}=\int_{t_{n}}^{t_{n+1}}\left(\begin{array}{cc}-L_{h}^{(1)}(s) & 0 \\ 0 & -L_{h}^{(1)}(s)\end{array}\right) d s$.

Therefore,

$$
\left\|\left(\mathscr{L}_{n+1}-\mathscr{L}_{n}\right) \Phi\right\|_{n+1} \leqslant \int_{t_{n}}^{t_{n+1}}\|\| \mathscr{L}_{h}^{(1)}(s) \mathscr{T}_{n} \mathscr{L}_{n} \Phi \|_{n+1} d s
$$

and

$$
\left\|\left(\mathscr{L}_{n+1}^{2}-\mathscr{L}_{n}^{2}\right) \Phi\right\|_{n+1} \leqslant \int_{t_{n}}^{t_{n+1}}\left\|\left(\begin{array}{cc}
-L_{h}^{(1)}(s) & 0 \\
0 & -L_{h}^{(1)}(s)
\end{array}\right) \mathscr{T}_{n}^{2} \mathscr{L}_{n}^{2} \Phi\right\|_{n+1} d s
$$

Since

$$
\left\|\mathscr{L}_{h}^{(1)}(s) \mathscr{T}_{n} \psi\right\|\left\|_{n+1}=\right\| T_{n+1}^{1 / 2} L_{h}^{(1)}(s) T_{n} \psi_{2}\|\leqslant C\| T_{n+1}^{1 / 2} \psi_{2} \|
$$

and

$$
\begin{aligned}
& \left\|\left(\begin{array}{cc}
-L_{h}^{(1)}(s) & 0 \\
0 & -L_{h}^{(1)}(s)
\end{array}\right) \mathscr{T}_{n}^{2} \psi \mid\right\|_{n+1}^{2}=\left\|L_{h}^{(1)}(s) T_{n} \psi_{1}\right\|^{2}+\left\|T_{n+1}^{1 / 2} L_{h}^{(1)}(s) T_{n} \psi_{2}\right\|^{2} \\
& \leqslant C\left(\left\|\psi_{1}\right\|^{2}+\left\|T_{n+1}^{1 / 2} \psi_{2}\right\|^{2}\right),
\end{aligned}
$$

it follows that

$$
\left\|k\left(\mathscr{L}_{n+1}-\mathscr{L}_{n}\right) \Phi\right\|_{n+1} \leqslant C k^{2}\|\| \mathscr{L}_{n} \Phi \|_{n+1}
$$

and

$$
\left\|k^{2}\left(\mathscr{L}_{n+1}^{2}-\mathscr{L}_{n}^{2}\right) \Phi\right\|_{n+1} \leqslant C k^{3}\left\|\mathscr{L}_{n}^{2} \Phi\right\|_{n+1}
$$


These two inequalities used together with (4.27) and Lemma 4.2 imply (4.30) and (4.31). Substitution of $\Phi=E^{n}$ in (4.30) and (4.31) and the triangle inequality give (4.28) and (4.29).

LEMMA 4.6.

$$
\left\|\left(\tilde{P}_{n}-P_{n}\right) E^{n}\right\|_{n+1} \leqslant C k\left\|Q_{n} E^{n}\right\|_{n}
$$

and

$$
\left\|\left(Q_{n+1}-\tilde{Q}_{n+1}\right) E^{n+1}\right\|_{n+1} \leqslant C k\left\|Q_{n+1} E^{n+1}\right\|_{n+1} .
$$

Proof. The proof is the same as the one given in Lemma 4.3. If $p(x)$ is quadratic, then

$$
\begin{aligned}
\left\|\left(\tilde{P}_{n}-P_{n}\right) E^{n}\right\|_{n+1} & =\left\|p_{2} k^{2} \mathscr{L}_{n}^{(1)} E^{n}\right\|_{n+1} \leqslant C k^{2}\left\|\mathscr{L}_{n}^{(1)} \mathscr{T}_{n} \mathscr{L}_{n} E^{n}\right\|_{n} \\
& \leqslant C k\left\|Q_{n} E^{n}\right\|_{n},
\end{aligned}
$$

where we have used (4.27) and the proof of Lemma 4.3. (4.35) follows from almost exactly the same argument.

LEMMA 4.7. The "truncation error"

$$
\left\|\tilde{Q}_{n+1} U_{h}\left(t_{n+1}\right)-\tilde{P}_{n} U_{h}\left(t_{n}\right)\right\|_{n+1} \leqslant C k^{\nu} \int_{t_{n}}^{t_{n+1}}\left\|U_{h}^{(\nu+1)}(s)\right\|_{n+1} d s .
$$

Proof. By definition

$$
\begin{aligned}
\tilde{Q}_{n+1} U_{h}\left(t_{n+1}\right)-\tilde{P}_{n} U_{h}\left(t_{n}\right) & \\
= & U_{h}\left(t_{n+1}\right)+q_{1} k U_{h}^{(1)}\left(t_{n+1}\right)+q_{2} k^{2} U_{h}^{(2)}\left(t_{n+1}\right) \\
& -U_{h}\left(t_{n}\right)-p_{1} k U_{h}^{(1)}\left(t_{n}\right)-p_{2} k^{2} U_{h}^{(2)}\left(t_{n}\right) .
\end{aligned}
$$

The inequality (4.36) follows from this equality and Lemma 4.1.

The next theorem uses Lemmas 4.4, 4.5, 4.6 and 4.7 to estimate the terms appearing in the error equation (4.23). The fully discrete error estimate is the result.

THEOREM 4.1. The error $E^{n}=U_{h}\left(t_{n}\right)-W^{n}$ satisfies the estimate

$$
\begin{aligned}
& \|\| Q_{n+1} E^{n+1} \|_{n+1}^{2} \\
& \quad \leqslant(1+C k)\left\|Q_{n} E^{n}\right\|_{n}^{2}+C k^{2 \nu-1}\left(\int_{t_{n}}^{t_{n+1}}\left\|U_{h}^{(\nu+1)}(s)\right\| \|_{n+1} d s\right)^{2},
\end{aligned}
$$

for $n=0,1, \ldots, N-1$, and

$$
\left\|Q_{N} E^{N}\right\|_{N} \leqslant C\left\|Q_{0} E^{0}\right\|_{0}+C k^{\nu}\left(\sum_{j=0}^{\nu+1}\left\|U_{h}^{(j)}(0)\right\|_{0}\right) .
$$

Proof. To prove (4.37) we take the $((\cdot, \cdot))_{n+1}$ inner product of (4.23) with $Q_{n+1} E^{n+1}$ and use Lemmas 4.4, 4.5, 4.6, and 4.7 to estimate the terms appearing after the equality in (4.23). These terms are estimated as follows:

(1)

$$
\begin{aligned}
\left(\left(P_{n+1} E^{n}, Q_{n+1} E^{n+1}\right)\right)_{n+1} & \leqslant\left\|P_{n+1} E^{n}\right\|\left\|_{n+1}\right\| Q_{n+1} E^{n+1}\|\|_{n+1} \\
& \leqslant \frac{1}{2}\left\|P_{n+1} E^{n}\right\|_{n+1}^{2}+\frac{1}{2}\left\|Q_{n+1} E^{n+1}\right\|_{n+1}^{2}
\end{aligned}
$$


Using Lemma 4.4, it follows that

$$
\left(\left(P_{n+1} E^{n}, Q_{n+1} E^{n+1}\right)\right)_{n+1} \leqslant \frac{1}{2}(1+C k)\left\|Q_{n} E^{n}\right\|_{n}^{2}+\frac{1}{2}\left\|Q_{n+1} E^{n+1}\right\|_{n+1}^{2} .
$$

$$
\left(\left(\left(P_{n}-P_{n+1}\right) E^{n}, Q_{n+1} E^{n+1}\right)\right)_{n+1} \leqslant\left\|\left(P_{n}-P_{n+1}\right) E^{n}\right\|_{n+1}\left\|Q_{n+1} E^{n+1}\right\|_{n+1} .
$$

Using (4.28) in Lemma 4.5 gives

$$
\begin{aligned}
\left(\left(\left(P_{n}-P_{n+1}\right) E^{n}, Q_{n+1} E^{n+1}\right)\right)_{n+1} & \leqslant C k\left(\left\|Q_{n} E^{n}\right\|\left\|_{n}\right\| Q_{n+1} E^{n+1}\|\|_{n+1}\right) \\
& \leqslant \frac{C}{2} k\left(\left\|Q_{n} E^{n}\right\|_{n}^{2}+\left\|Q_{n+1} E^{n+1}\right\|_{n+1}^{2}\right) .
\end{aligned}
$$

(3) From Lemma (4.6)

$$
\left(\left(\left(\tilde{P}_{n}-P_{n}\right) E^{n}, Q_{n+1} E^{n+1}\right)\right)_{n+1} \leqslant C k\left(\left\|Q_{n} E^{n}\right\|_{n}^{2}+\left\|Q_{n+1} E^{n+1}\right\|_{n+1}^{2}\right)
$$

and

$$
\left(\left(\left(Q_{n+1}-\tilde{Q}_{n+1}\right) E^{n+1}, Q_{n+1} E^{n+1}\right)\right)_{n+1} \leqslant C k\left\|Q_{n+1} E^{n+1}\right\|_{n+1}^{2} .
$$

(4) From Lemma (4.7)

$$
\begin{aligned}
& \left(\left(\tilde{Q}_{n+1} U_{h}\left(t_{n+1}\right)-\tilde{P}_{n} U_{h}\left(t_{n}\right), Q_{n+1} E^{n+1}\right)\right)_{n+1} \\
& \quad \leqslant C\left(k^{\nu} \int_{t_{n}}^{t_{n+1}}\left\|U_{h}^{(\nu+1)}(s)\right\| \|_{n+1} d s\right)\|\| Q_{n+1} E^{n+1} \|_{n+1} \\
& \quad \leqslant C k^{2 \nu-1}\left(\int_{t_{n}}^{t_{n+1}}\left\|U_{h}^{(\nu+1)}(s)\right\|_{n+1} d s\right)^{2}+C k\left\|Q_{n+1} E^{n+1}\right\|_{n+1}^{2} .
\end{aligned}
$$

Using the estimates (4.39)-(4.42) and (4.43), gives

$$
\begin{aligned}
\left\|Q_{n+1} E^{n+1}\right\|_{n+1}^{2} \leqslant & \frac{1}{2}(1+C k)\left\|Q_{n} E^{n}\right\|_{n}^{2}+\frac{1}{2}\left\|Q_{n+1} E^{n+1}\right\|_{n+1}^{2} \\
& +C_{1} k\left\|Q_{n} E^{n}\right\|_{n}^{2}+C_{2} k\left\|Q_{n+1} E^{n+1}\right\|_{n+1}^{2} \\
& +C_{3} k^{2 \nu-1}\left(\int_{t_{n}}^{t_{n+1}}\left\|U_{h}^{(\nu+1)}(s)\right\|_{n+1} d s\right)^{2} .
\end{aligned}
$$

Subtracting the terms containing $\left\|Q_{n+1} E^{n+1} \mid\right\|_{n+1}^{2}$ on the right-hand side gives

$$
\begin{aligned}
\left(\frac{1}{2}-C_{2} k\right)\left\|Q_{n+1} E^{n+1}\right\|_{n+1}^{2} \leqslant & \left(\frac{1}{2}+\left(\frac{C}{2}+C_{1}\right) k\right)\left\|Q_{n} E^{n}\right\|_{n}^{2} \\
& +C_{3} k^{2 \nu-1}\left(\int_{t_{n}}^{t_{n+1}}\left\|U_{h}^{(\nu+1)}(s)\right\| \|_{n+1} d s\right)^{2} .
\end{aligned}
$$

If $k$ is small enough, dividing by $\left(\frac{1}{2}-C_{2} k\right)$ and bounding the constants produces (4.37). 
To prove (4.38), we start with (4.37). From Theorem 3.1 it follows that

$$
\begin{aligned}
\left(T_{h} u_{h}^{(\nu+2)}, u_{h}^{(\nu+2)}\right)(s)+\left\|u_{h}^{(\nu+1)}(s)\right\|^{2} \\
\leqslant C\left(\sum_{j=0}^{\nu+1}\left\|u_{h}^{(j)}(0)\right\|^{2}+\sum_{j=1}^{\nu+2}\left(T_{h} u_{h}^{(j)}, u_{h}^{(j)}\right)(0)\right) \\
\leqslant C \sum_{j=0}^{\nu+1}\left\|U_{h}^{(j)}(0)\right\|_{0}^{2} .
\end{aligned}
$$

It follows that

$$
\left(\int_{t_{n}}^{t_{n+1}}\left\|U^{(\nu+1)}(s)\right\|_{n+1} d s\right)^{2} \leqslant C k^{2} \sum_{j=0}^{\nu+1}\|\| U_{h}^{(j)}(0) \|_{0}^{2} .
$$

So (4.37) becomes

$$
\left\|Q_{n+1} E^{n+1}\right\|_{n+1}^{2} \leqslant(1+C k)\left\|Q_{n} E^{n}\right\|_{n}^{2}+C k^{2 \nu+1} \sum_{j=0}^{\nu+1}\left\|U_{h}^{(j)}(0)\right\|_{0}^{2} .
$$

Since $1+C k \leqslant e^{C k}$, the above estimate gives

$$
\left\|Q_{n+1} E^{n+1}\right\|_{n+1}^{2}-e^{C k}\left\|Q_{n} E^{n}\right\|_{n}^{2} \leqslant C k^{2 v+1} \sum_{j=0}^{\nu+1}\left\|U_{h}^{(j)}(0)\right\|_{0}^{2} .
$$

Muliiplying by $e^{-C t_{n+1}}$ yields

$$
e^{-C t_{n+1}}\left\|Q_{n+1} E^{n+1}\right\|_{n+1}^{2}-e^{-C t_{n}}\left\|Q_{n} E^{n}\right\|_{n}^{2} \leqslant C\left(k e^{-C t_{n+1}}\right)\left(k^{2 v}\right) \sum_{j=0}^{\nu+1}\left\|U_{h}^{(j)}(0)\right\|_{0}^{2} .
$$

Summing from $n=0$ to $N-1$ gives

$$
e^{-C t_{N}}\left\|Q_{N} E^{N}\right\|_{N}^{2}-\left\|Q_{0} E^{0}\right\| \leqslant C k^{2 \nu} \sum_{j=0}^{\nu+1}\left\|U_{h}^{(j)}(0)\right\|_{0}^{2}
$$

or

$$
\left\|Q_{N} E^{N}\right\|_{N}^{2} \leqslant e^{C t_{N}}\left\|Q_{0} E^{0}\right\|_{0}^{2}+C\left(e^{C t_{N}}\right)\left(k^{2 \nu}\right) \sum_{j=0}^{\nu+1}\left\|U_{h}^{(j)}(0)\right\|_{0}^{2} .
$$

Taking the square root and defining new constants produces (4.38). This completes the proof of the theorem.

Theorem 4.1 and Lemma 4.2 imply that

$$
\left\|E^{N}\right\|_{N} \leqslant C\left\|Q_{0} E^{0}\right\|_{0}+C k^{\nu}\left(\sum_{j=0}^{\nu+1}\left\|U_{h}^{(j)}(0)\right\|_{0}\right) .
$$

Since $\left\|u_{h}\left(t_{N}\right)-W_{1}^{N}\right\| \leqslant\left\|E^{N}\right\|_{N}$, it follows that

$$
\left\|u_{h}\left(t_{N}\right)-W_{1}^{N}\right\| \leqslant C\left\|Q_{0} E^{0}\right\|_{0}+C k^{\nu}\left(\sum_{j=0}^{\nu+1}\left\|U_{h}^{(j)}(0)\right\|_{0}\right) .
$$

(4.44) and Theorem $3.2\left(U(t) \equiv\left(\begin{array}{c}u(t) \\ u_{t}(t)\end{array}\right)\right)$ imply that

$$
\begin{aligned}
\left\|u\left(t_{N}\right)-W_{1}^{N}\right\| \leqslant C( & \left\|U(0)-U_{h}(0)\right\|_{0}+h^{s}\left(\left\|u^{0}\right\|_{s+1}+\left\|u_{t}^{0}\right\|_{s}\right) \\
& \left.+\left\|Q_{0}\left(U_{h}(0)-W^{0}\right)\right\|_{0}+k^{\nu} \sum_{j=0}^{\nu+1}\left\|U_{h}^{(j)}(0)\right\|_{0}\right)
\end{aligned}
$$


for $2 \leqslant s \leqslant r$. It remains to be shown that three of the four terms on the right-hand side of (4.45) can be bounded in an appropriate way so that (4.45) is an optimal error estimate. Optimal is defined as the type of estimate derived in Baker and Bramble [2], where the case of time-independent coefficients was considered. Bounding the terms in (4.45) requires special choices for $U_{h}(0)$ and $W^{0}$ so that (4.45) will be an optimal estimate. The construction of functions which we will use for $U_{h}(0)$ and $W^{0}$ is the subject of the next section.

5. Construction of Initial Data $U_{h}(0)$. In this section operators on $L^{2} \times L^{2}$ are constructed out of $\mathscr{L}^{(j)}$ and $\mathscr{T}^{(j)}$ and operators on $S_{h} \times S_{h}$ out of $\mathscr{L}_{h}^{(j)}$ and $\mathscr{T}_{h}^{(j)}$. These operators are analogous to operators used for parabolic equations with time-dependent coefficients by Sammon in [20] and [21]. They will be used to define special initial data for the semidiscrete problem as in the parabolic case. In Section 6 , we will show that with a special choice for $W^{0}$ the fully discrete scheme defined by (4.9) is not changed by the special initial data for the semidiscrete problem. Specifically, the fully discrete scheme (4.9) and $W^{0}$ do not depend on the parameter $\alpha$ which will be introduced below in order to construct the operators mentioned above.

With $U \equiv\left(\begin{array}{c}u \\ u_{t}\end{array}\right)$, the hyperbolic problem (1.1) can be written as the first order system

$$
U_{t}=\mathscr{L} U, \quad U(0)=\left(\begin{array}{l}
u^{0} \\
u_{t}^{0}
\end{array}\right)
$$

where $\mathscr{L} \equiv\left(\begin{array}{cc}0 & I \\ -L & 0\end{array}\right) . \mathscr{L}$ is an unbounded operator on $L^{2} \times L^{2}$ with domain $D_{\mathscr{L}}=$ $D_{L} \times L^{2}$. Also, with $\hat{U} \equiv e^{-\alpha t} U$, it follows that

$$
U_{t}=\alpha e^{\alpha t} \hat{U}+e^{\alpha t} \hat{U}_{t}
$$

and, using (5.1), that

$$
\hat{U}_{t}=(\mathscr{L}-\alpha I) \hat{U}, \quad \hat{U}(0)=U(0)=\left(\begin{array}{c}
u^{0} \\
u_{t}^{0}
\end{array}\right) .
$$

Differentiation of (5.2) $m$ times gives

$$
\hat{U}^{(m+1)}=\hat{A}_{m+1} \hat{U}
$$

where $\hat{A_{0}} \equiv I$, for $0 \leqslant j \leqslant m$,

$$
\hat{A}_{j+1} \equiv \sum_{i=0}^{j}\left(\begin{array}{l}
j \\
i
\end{array}\right) \hat{\mathscr{L}}^{(j-i)} \hat{A}_{i}
$$

and $\hat{\mathscr{L}}^{(j-i)}$ is the $(j-i)$ time derivative of $\hat{\mathscr{L}} \equiv \mathscr{L}-\alpha I . \hat{A}_{m+1}$ is an unbounded operator with domain $D\left(\hat{A}_{m+1}\right)=\left\{V \in L^{2} \times L^{2}\right.$ such that $\hat{A}_{j} V \in D_{\mathscr{L}}$ for $j=$ $0, \ldots, m\}$.

For $0 \leqslant j \leqslant m+1$ the $j$ th time derivative of $\hat{\mathscr{T}}=(\hat{\mathscr{L}})^{-1}$ is denoted by $\hat{\mathscr{T}}^{(j)}$. We will show that if $\alpha$ is large enough then $\hat{A}_{m+1}$ is invertible and that $\hat{A}_{m+1}$ has properties which are similar to the properties of $(\hat{\mathscr{L}})^{m+1}$. (Note that if the coefficients of $L$ are independent of $t$ then $\hat{A}_{m+1}=(\hat{\mathscr{L}})^{m+1}$.)

THEOREM 5.1. Let $\hat{E}_{1}=\hat{\mathscr{L}}, \hat{E}_{2}=\hat{\mathscr{L}}\left(I-\hat{\mathscr{T}}^{(1)}\right)$, and for $m \geqslant 2$

$$
\hat{E}_{m+1}=\hat{\mathscr{L}}\left(I-m \hat{\mathscr{T}}^{(1)}-\sum_{l=0}^{m-2}\left(\begin{array}{c}
m \\
l
\end{array}\right) \hat{\mathscr{T}}^{(m-l)} \hat{E}_{l+2}^{-1} \cdots \hat{E}_{m}^{-1}\right)
$$


(where $\hat{E}_{l}^{-1}$ is the inverse of $\hat{E}_{l}$ ). $\hat{E}_{l}$ is an unbounded operator on $L^{2} \times L^{2}$ with domain $D_{\mathscr{L}}$. The following conditions are satisfied for integers $l \geqslant 0$ and $m \geqslant 1$ :

(1) $\hat{E}_{1}, \ldots, \hat{E}_{m}:\left(H^{l+2} \cap H_{0}^{1}\right) \times H^{l+1} \rightarrow H^{l+1} \times H^{l}$ exist and are bounded.

(2) $\hat{E}_{1}^{-1}, \ldots, \hat{E}_{m}^{-1}: H^{l+1} \times H^{l} \rightarrow\left(H^{l+2} \cap H_{0}^{1}\right) \times H^{l+1}$ exist and are bounded.

(3) $\hat{A}_{m}=\hat{E}_{m} \cdots \hat{E}_{1}$ on $D\left(\hat{A}_{m}\right)$.

The next result is an analogue of Theorem 5.1 for operators on $S_{h} \times S_{h}$ which approximate the operators $\hat{E}_{1}^{-1}, \ldots, \hat{E}_{m}^{-1}$ and $\hat{A}_{m}^{-1}$. Analogous to (5.3), the derivatives of $\hat{U}_{h}^{(1)}=\hat{\mathscr{L}}_{h} \hat{U}_{h}$ can be written

$$
\hat{U}_{h}^{(m+1)}=\hat{A}_{m+1, h} \hat{U}_{h}
$$

where $\hat{A_{0, h}} \equiv I$, for $0 \leqslant j \leqslant m$,

$$
\hat{A}_{j+1, h}=\sum_{i=0}^{j}\left(\begin{array}{l}
j \\
i
\end{array}\right) \hat{\mathscr{L}}_{h}^{(j-i)} \hat{A}_{i, h},
$$

and $\hat{\mathscr{L}}_{h}^{(j-i)}$ is the $(j-i)$ time derivative of $\hat{\mathscr{L}}_{h} \equiv \mathscr{L}_{h}-\alpha I$. For $1 \leqslant j \leqslant m+1$ the $j$ th time derivative of $\hat{\mathscr{T}}_{h} \equiv\left(\hat{\mathscr{L}}_{h}\right)^{-1}$ is denoted by $\hat{\mathscr{T}}_{h}^{(j)}$.

THEOREM 5.2. For $\alpha$ sufficiently large the operators $\hat{E}_{1, h}=\hat{\mathscr{L}}_{h}, \hat{E}_{2, h}=\hat{\mathscr{L}}_{h}\left(I-\hat{\mathscr{T}}_{h}^{(1)}\right)$ and, for $m \geqslant 2$,

$$
\hat{E}_{m+1, h}=\hat{\mathscr{L}}_{h}\left(I-m \hat{\mathscr{T}}_{h}^{(1)}-\sum_{l=0}^{m-2}\left(\begin{array}{c}
m \\
l
\end{array}\right) \hat{\mathscr{T}}_{h}^{(m-l)} \hat{E}_{l+2, h}^{-1} \cdots \hat{E}_{m, h}^{-1}\right)
$$

are invertible on $S_{h} \times S_{h}$; for $m \geqslant 1$ and $2 \leqslant s \leqslant r$,

and for $F=\left(\begin{array}{c}f_{1} \\ f_{2}\end{array}\right) \in H^{s-2} \times H^{s-2}$

$$
\hat{A}_{m, h}=\hat{E}_{m, h} \cdots \hat{E}_{1, h} \text { on } S_{h} \times S_{h}
$$

$$
\left\|\left(\hat{E}_{m}^{-1}-\hat{E}_{m, h}^{-1}\right) F\right\|_{0} \leqslant C(\alpha) h^{s}\left(\left\|f_{1}\right\|_{s-2}+\left\|f_{2}\right\|_{s-2}\right),
$$

where $\hat{E}_{m, h}^{-1}$ are extended to $L^{2} \times L^{2}$ by the formula $\hat{E}_{m, h}^{-1} \equiv \hat{E}_{m, h}^{-1}\left(\begin{array}{ll}P & 0 \\ 0\end{array}\right)$ (where $P$ is the $L^{2}$ orthogonal projection onto $S_{h}$ ).

Remark. The seminorm $\mid\|\cdot\|_{0}$ used in (5.9) can be replaced by the seminorm

$$
\left(\left\|\phi_{1}\right\|^{2}+\left(T_{h}(t) \phi_{2}, \phi_{2}\right)\right)^{1 / 2} \text { for }\left(\begin{array}{l}
\phi_{1} \\
\phi_{2}
\end{array}\right) \in L^{2} \times L^{2}
$$

and for any $t \in[0, \tau]$ since these seminorms are equivalent (see (3.16)). We use III $\cdot \|_{0}$ since the operators $\hat{E}_{1}, \ldots, \hat{E}_{m}$ and $\hat{E}_{1, h}, \ldots, \hat{E}_{m, h}$ will only be used at $t=0$ to construct the special initial data for the semidiscrete problem.

The proofs of Theorems 5.1 and 5.2 are in Section 8 which appears in the supplements section of this issue.

6. $L^{2}$ Error Estimates. In this section we combine the error estimates of Section 3 and Section 4 using a special choice for $U_{h}(0)$ which is based on the operators constructed in Section 5. In Sections 2, 3, and 4 the following three equations were defined and error estimates were derived which did not include estimates for the initial data.

The hyperbolic equation:

$$
U_{t}=\mathscr{L} U, \quad t \in[0, \tau], \quad U(0)=\left(\begin{array}{c}
u^{0} \\
u_{t}^{0}
\end{array}\right)
$$


The semidiscrete equation:

$$
\left(U_{h}\right)_{t}=\mathscr{L}_{h} U_{h}, \quad t \in[0, \tau], \quad\left(U_{h}\right)(0) \equiv \hat{A}_{m, h}^{-1}(0) \hat{A}_{m}(0)\left(\begin{array}{c}
u^{0} \\
u_{t}^{0}
\end{array}\right) .
$$

The fully discrete equation:

$$
\begin{gathered}
\tilde{Q}_{n+1} W^{n+1}=\tilde{P}_{n} W^{n}, \quad n=0,1, \ldots, N-1, \\
W^{0} \equiv \tilde{Q}_{0}^{-1}\left(\begin{array}{cc}
P & 0 \\
0 & P
\end{array}\right)\left(I+q_{1} k \mathscr{L}(0)+q_{2} k^{2}\left(\mathscr{L}^{2}(0)+\mathscr{L}^{(1)}(0)\right)\right)\left(\begin{array}{c}
u^{0} \\
u_{t}^{0}
\end{array}\right),
\end{gathered}
$$

where $m$ is an integer greater than or equal to $\nu+1$ and $P$ is the $L^{2}$ orthogonal projection onto $S_{h}$.

The functions $u_{j}$ defined by

$$
u_{0}=u^{0}, \quad u_{1}=u_{t}^{0}, \quad u_{j}=-\sum_{k=0}^{j-2}\left(\begin{array}{c}
j-2 \\
k
\end{array}\right) L^{(j-2-k)}(0) u_{k} \quad \text { for } j \geqslant 2,
$$

were introduced in Section 2 where assumptions on $u_{j}$ implied existence of time derivatives of $u$. These assumptions were that $u_{j} \in D_{L}=H^{2} \cap H_{0}^{1}$. The three lemmas in this section contain estimates for the error in the initial data for the semidiscrete equation and for the fully discrete equation, as well as a bound for the time derivatives of the solution of the semidiscrete equation. The following lemma contains the error estimate for the initial data for the semidiscrete equation.

LEMMA 6.1. If $u_{j} \in D_{L}$ for $j=0, \ldots, m+s-3$ and $u_{m+s-2} \in H_{0}^{1}$, then for $2 \leqslant$ $s \leqslant r$ and $j=0, \ldots, m-1$,

$$
\left\|U^{(j)}(0)-U_{h}^{(j)}(0)\right\|_{0} \leqslant C h^{s}\left(\left\|u^{0}\right\|_{m+s-1}+\left\|u_{t}^{0}\right\|_{m+s-2}\right) .
$$

Proof. Since $\hat{U}=e^{-\alpha t} U$ and $\hat{U}_{h}=e^{-\alpha t} U_{h}, \hat{U}(0)=U(0), \hat{U}_{h}(0)=U_{h}(0)$ and

$$
U^{(j)}(0)-U_{h}^{(j)}(0)=\sum_{l=0}^{j}\left(\begin{array}{l}
j \\
l
\end{array}\right)(\alpha)^{j-l}\left(\hat{U}_{h}^{(l)}(0)-\hat{U}_{h}^{(l)}(0)\right) .
$$

Therefore,

$$
\left\|U^{(j)}(0)-U_{h}^{(j)}(0)\right\|_{0} \leqslant C \sum_{l=0}^{j}\left\|\hat{U}^{(l)}(0)-\hat{U}_{h}^{(l)}(0)\right\|_{0} .
$$

Using (5.3),

$$
\hat{U}^{(l)}(0)=\hat{A}_{l}(0) \hat{U}(0)=\hat{A}_{l}(0) \hat{A}_{m}^{-1}(0) \hat{A}_{m}(0)\left(\begin{array}{l}
u^{0} \\
u_{t}^{0}
\end{array}\right)
$$

and from (5.6) and (6.2)

$$
\hat{U}_{h}^{(l)}(0)=\hat{A}_{l, h}(0) \hat{U}_{h}(0)=\hat{A}_{l, h}(0) \hat{A}_{m, h}^{-1}(0) \hat{A}_{m}(0)\left(\begin{array}{c}
u^{0} \\
u_{t}^{0}
\end{array}\right) .
$$

Theorems 5.1 and 5.2 imply that

$$
\begin{aligned}
\hat{U}^{(l)}(0)-\hat{U}_{h}^{(l)}(0) & =\left(\hat{A_{l}}(0) \hat{A}_{m}^{-1}(0)-\hat{A}_{l, h}(0) \hat{A}_{m, h}^{-1}(0)\right) \hat{A}_{m}(0)\left(\begin{array}{c}
u^{0} \\
u_{t}^{0}
\end{array}\right) \\
& =\left(\hat{E}_{l+1}^{-1} \cdots \hat{E}_{m}^{-1}-\hat{E}_{l+1, h}^{-1} \cdots \hat{E}_{m, h}^{-1}\right)(0) \hat{A}_{m}(0)\left(\begin{array}{c}
u^{0} \\
u_{t}^{0}
\end{array}\right)
\end{aligned}
$$


Since

$$
\begin{aligned}
\hat{E}_{l+1}^{-1} \cdots \hat{E}_{m}^{-1}- & \hat{E}_{l+1, h}^{-1} \cdots \hat{E}_{m, h}^{-1} \\
& =\sum_{i=l+1}^{m} \hat{E}_{l+1, h}^{-1} \cdots \hat{E}_{i-1, h}^{-1}\left(\hat{E}_{i}^{-1}-\hat{E}_{i, h}^{-1}\right) \hat{E}_{i+1}^{-1} \cdots \hat{E}_{m}^{-1}
\end{aligned}
$$

and

$$
\begin{aligned}
\hat{A}_{m}(0)\left(\begin{array}{c}
u^{0} \\
u_{t}^{0}
\end{array}\right) & =\hat{U}^{(m)}(0)=\sum_{l=0}^{m}\left(\begin{array}{c}
m \\
l
\end{array}\right)(-\alpha)^{m-l} U^{(l)}(0) \\
& =\sum_{l=0}^{m}\left(\begin{array}{c}
m \\
l
\end{array}\right)(-\alpha)^{m-l}\left(\begin{array}{c}
u_{l} \\
u_{l+1}
\end{array}\right),
\end{aligned}
$$

it follows from Theorems 5.1 and 5.2 that

$$
\left\|U^{(j)}(0)-U_{h}^{(j)}(0)\right\|_{0} \leqslant C h^{s} \sum_{l=0}^{m}\left(\left\|u_{l}\right\|_{s-2}+\left\|u_{l+1}\right\|_{s-2}\right) .
$$

(6.4) follows from this inequality because from (2.15)

$$
\sum_{l=0}^{m}\left(\left\|u_{l}\right\|_{s-2}+\left\|u_{l+1}\right\|_{s-2}\right) \leqslant C\left(\left\|u^{0}\right\|_{m+s-1}+\left\|u_{t}^{0}\right\|_{m+s-2}\right) .
$$

The next lemma contains a bound for the time derivatives at $t=0$ of the solution of the semidiscrete equation.

LEMMA 6.2. If $u_{j} \in D_{L}$ for $j=0, \ldots, m-1$ and $u_{m} \in H_{0}^{1}$, then for $j=0, \ldots, m$,

$$
\left\|U_{h}^{(j)}(0)\right\|_{0} \leqslant C\left(\left\|u^{0}\right\|_{m+1}+\left\|u_{t}^{0}\right\|_{m}\right) .
$$

Proof. Since $U_{h}=e^{\alpha t} \hat{U}_{h}$,

$$
U_{h}^{(j)}(0)=\sum_{l=0}^{j}\left(\begin{array}{l}
j \\
l
\end{array}\right) \alpha^{(j-l)} \hat{U}_{h}^{(l)}(0) .
$$

Also, from (5.6) and (6.2)

$$
\hat{U}_{h}^{(l)}(0)=\hat{A}_{l, h}(0) \hat{U}_{h}(0)=\hat{A}_{l, h}(0) \hat{A}_{m, h}^{-1}(0) \hat{A}_{m}(0)\left(\begin{array}{c}
u^{0} \\
u_{t}^{0}
\end{array}\right) .
$$

Using Theorem 5.2

$$
\hat{U}_{h}^{(l)}(0)=\left(\hat{E}_{l+1, h}^{-1} \cdots \hat{E}_{m, h}^{-1}\right)(0) \hat{A}_{m}(0)\left(\begin{array}{l}
u^{0} \\
u_{t}^{0}
\end{array}\right) .
$$

(8.36) implies that

$$
\left\|\hat{U}_{h}^{(l)}(0)\right\|_{0} \leqslant C\left\|\hat{A}_{m}(0)\left(\begin{array}{c}
u^{0} \\
u_{t}^{0}
\end{array}\right)\right\| \|_{0}
$$

and (6.6) follows from this estimate.

The following lemma is the error estimate for the initial data for the fully discrete equation.

Lemma 6.3. If $u_{j} \in D_{L}$ for $j=0, \ldots, m+s-3$ and $u_{m+s-2} \in H_{0}^{1}$, then for $2 \leqslant$ $s \leqslant r$,

$$
\left\|\tilde{Q}_{0}\left(U_{h}(0)-W^{0}\right)\right\|_{0} \leqslant C h^{s}\left(\left\|u^{0}\right\|_{m+s-1}+\left\|u_{t}^{0}\right\|_{m+s-2}\right) .
$$


Proof. By definition

$$
\tilde{Q}_{0} W^{0}=\left(\begin{array}{cc}
P & 0 \\
0 & P
\end{array}\right)\left(I+q_{1} k \mathscr{L}(0)+q_{2} k^{2}\left(\mathscr{L}^{2}(0)+\mathscr{L}^{(1)}(0)\right)\right)\left(\begin{array}{l}
u^{0} \\
u_{t}^{0}
\end{array}\right)
$$

and

$$
\tilde{Q}_{0} U_{h}(0)=\left(I+q_{1} k \mathscr{L}_{0}+q_{2} k^{2}\left(\mathscr{L}_{0}^{2}+\mathscr{L}_{0}^{(1)}\right)\right) U_{h}(0)
$$

Hence

$$
\begin{aligned}
& \tilde{Q}_{0}\left(W^{0}-U_{h}(0)\right) \\
& =\left(\begin{array}{ll}
P & 0 \\
0 & P
\end{array}\right)\left(I+q_{1} k(\mathscr{L}(0)-\alpha I)+q_{2} k^{2}\left((\mathscr{L}(0)-\alpha I)^{2}+\mathscr{L}^{(1)}(0)\right)\right)\left(\begin{array}{l}
u^{0} \\
u_{t}^{0}
\end{array}\right) \\
& -\left(I+q_{1} k\left(\mathscr{L}_{0}-\alpha I\right)+q_{2} k^{2}\left(\left(\mathscr{L}_{0}-\alpha I\right)^{2}+\mathscr{L}_{0}^{(1)}\right)\right) U_{h}(0) \\
& -\left(\begin{array}{ll}
P & 0 \\
0 & P
\end{array}\right)\left(-q_{1} k \alpha I+q_{2} k^{2}\left(-2 \alpha \mathscr{L}(0)+\alpha^{2} I\right)\right)\left(\begin{array}{l}
u^{0} \\
u_{t}^{0}
\end{array}\right) \\
& +\left(-q_{1} k \alpha I+q_{2} k^{2}\left(-2 \alpha \mathscr{L}_{0}+\alpha^{2} I\right)\right) U_{h}(0) \\
& =\left(\begin{array}{cc}
P & 0 \\
0 & P
\end{array}\right)\left(\left(1+q_{1} k \alpha+q_{2} k^{2} \alpha^{2}\right) I+q_{1} k \hat{\mathscr{L}}(0)\right. \\
& \left.+q_{2} k^{2}\left(\hat{\mathscr{L}}^{2}(0)+\hat{\mathscr{L}}^{(1)}(0)\right)\right)\left(\begin{array}{l}
u^{0} \\
u_{t}^{0}
\end{array}\right) \\
& -\left(\left(1+q_{1} k \alpha+q_{2} k^{2} \alpha^{2}\right) I+q_{1} k \hat{\mathscr{L}}_{0}+q_{2} k^{2}\left(\hat{\mathscr{L}}_{0}^{2}+\hat{\mathscr{L}}_{0}^{(1)}\right)\right) U_{h}(0) \\
& -\left(\begin{array}{cc}
P & 0 \\
0 & P
\end{array}\right)\left(-2 q_{2} k^{2} \alpha(\mathscr{L}(0)-\alpha I)\right)\left(\begin{array}{l}
u^{0} \\
u_{t}^{0}
\end{array}\right)+\left(-2 q_{2} k^{2} \alpha\left(\mathscr{L}_{0}-\alpha I\right)\right) U_{h}(0) \\
& =\left(\begin{array}{ll}
P & 0 \\
0 & P
\end{array}\right)\left(\left(1+q_{1} k \alpha+q_{2} k^{2} \alpha^{2}\right) I+\left(q_{1} k+2 q_{2} k^{2} \alpha\right) \hat{\mathscr{L}}(0)\right. \\
& \left.+q_{2} k^{2}\left(\hat{\mathscr{L}}^{2}(0)+\hat{\mathscr{L}}^{(1)}(0)\right)\right)\left(\begin{array}{l}
u^{0} \\
u_{t}^{0}
\end{array}\right) \\
& -\left(\left(1+q_{1} k \alpha+q_{2} k^{2} \alpha^{2}\right) I+\left(q_{1} k+2 q_{2} k^{2} \alpha\right) \hat{\mathscr{L}}_{0}+q_{2} k^{2}\left(\hat{\mathscr{L}}_{0}^{2}+\hat{\mathscr{L}}_{0}^{(1)}\right)\right) U_{h}(0) .
\end{aligned}
$$

Therefore,

$$
\begin{aligned}
\tilde{Q}_{0}\left(W^{0}-U_{h}(0)\right)= & \left(1+q_{1} k \alpha+q_{2} k^{2} \alpha^{2}\right)\left[\left(\begin{array}{ll}
P & 0 \\
0 & P
\end{array}\right)\left(\begin{array}{l}
u^{0} \\
u_{t}^{0}
\end{array}\right)-U_{h}(0)\right] \\
& +\left(q_{1} k+2 q_{2} k^{2} \alpha\right)\left[\left(\begin{array}{ll}
P & 0 \\
0 & P
\end{array}\right) \hat{\mathscr{L}}(0)\left(\begin{array}{l}
u^{0} \\
u_{t}^{0}
\end{array}\right)-\hat{\mathscr{L}}_{0} U_{h}(0)\right] \\
& +q_{2} k^{2}\left(\left(\begin{array}{ll}
P & 0 \\
0 & P
\end{array}\right)\left(\hat{\mathscr{L}}^{2}(0)+\hat{\mathscr{L}}^{(1)}(0)\left(\begin{array}{l}
u^{0} \\
u_{t}^{0}
\end{array}\right)\right)-\left(\hat{\mathscr{L}}_{0}^{2}+\hat{\mathscr{L}}_{0}^{(1)}\right) U_{h}(0)\right) .
\end{aligned}
$$

Since $U_{h}(0)=\hat{U}_{h}(0)$,

$$
\begin{aligned}
\tilde{Q}_{0}\left(W^{0}-U_{h}(0)\right) \\
=\left(\begin{array}{rr}
P & 0 \\
0 & P
\end{array}\right)\left[\left(1+q_{1} k \alpha+q_{2} k^{2} \alpha^{2}\right)\left(\hat{U}(0)-\hat{U}_{h}(0)\right)\right. \\
\left.\quad+\left(q_{1} k+2 q_{2} k^{2} \alpha\right)\left(\hat{U}^{(1)}(0)-\hat{U}_{h}^{(1)}(0)\right)+q_{2} k^{2}\left(\hat{U}^{(2)}(0)-\hat{U}_{h}^{(2)}(0)\right)\right] .
\end{aligned}
$$


From (6.5) in the proof of Lemma 6.1 it follows that

$$
\left\|\tilde{Q}_{0}\left(W^{0}-U_{h}(0)\right)\right\|_{0} \leqslant C h^{s}\left(\left\|u^{0}\right\|_{m+s-1}+\left\|u_{t}^{0}\right\|_{m+s-2}\right) .
$$

This completes the proof of the lemma.

The next theorem combines the estimates in Lemmas 6.1, 6.2 and 6.3 with the semidiscrete error estimates (Theorem 3.2) and the fully discrete error estimate (Theorem 4.1).

THEOREM 6.1. Let $m$ be an integer such that $m \geqslant \nu+1$ and $\tau=N k$. If $u_{i} \in D_{L}$ for $i=0, \ldots, m+s-3$ and $u_{m+s-2} \in H_{0}^{1}$, then for $2 \leqslant s \leqslant r$ and $j=0,1, \ldots, m-2$,

$$
\begin{gathered}
\left\|U^{(j)}\left(t_{N}\right)-U_{h}^{(j)}\left(t_{N}\right)\right\|_{N} \leqslant C h^{s}\left(\left\|u^{0}\right\|_{m+s-1}+\left\|u_{t}^{0}\right\|_{m+s-2}\right), \\
\left\|Q_{N}\left(U_{h}\left(t_{N}\right)-W^{N}\right)\right\|_{N} \leqslant C\left(h^{s}+k^{\nu}\right)\left(\left\|u^{0}\right\|_{m+s-1}+\left\|u_{t}^{0}\right\|_{m+s-2}\right), \\
\left\|U_{h}\left(t_{N}\right)-W^{N}\right\|_{N} \leqslant C\left(h^{s}+k^{\nu}\right)\left(\left\|u^{0}\right\|_{m+s-1}+\left\|u_{t}^{0}\right\|_{m+s-2}\right),
\end{gathered}
$$

and

$$
\begin{aligned}
\left\|u\left(t_{N}\right)-W_{1}^{N}\right\|+ & \left\|T_{h}^{1 / 2}\left(u_{t}\left(t_{N}\right)-W_{2}^{N}\right)\right\| \\
& \leqslant C\left(h^{s}+k^{\nu}\right)\left(\left\|u^{0}\right\|_{m+s-1}+\left\|u_{t}^{0}\right\|_{m+s-2}\right) .
\end{aligned}
$$

Proof. (6.8) follows from (3.25) in Theorem $3.2(J=m-2)$ and Lemma 6.1. (6.9) follows from (4.38) in Theorem 4.1 and Lemmas 6.2 and 6.3. (6.10) follows from (6.9) and (4.16) (or (4.14) if $q_{2}=0$ ) in Lemma 4.2. (6.8), (6.10) and the triangle inequality imply (6.11). This completes the proof.

(6.11) shows that the error $u\left(t_{N}\right)-W_{1}^{N}$ is optimal in $L^{2} . W_{1}^{n}, n=0,1, \ldots, N$, is the first component of the solution $W^{n} \in S_{h} \times S_{h}$ of (6.3).

7. Examples of the Approximate Solution Operator $T_{h}$ for the Dirichlet Problem. These examples and more references are given in Bramble, Schatz, Thomée and Wahlbin [6].

(1) The "Standard" Galerkin Method. This is the example mentioned in Section 3. Here $S_{h} \subset H_{0}^{1}$ and is assumed to satisfy the approximation property

$$
\inf _{\chi \in S_{h}}\left\{\|w-\chi\|+h\|w-\chi\|_{1}\right\} \leqslant C h^{s}\|w\|_{s} \text { for } 1 \leqslant s \leqslant r .
$$

The operators $T_{h}: L^{2} \rightarrow S_{h}$ are defined by

$$
a\left(T_{h} f, \chi\right)=(f, \chi) \text { for } \chi \in S_{h} .
$$

(2) Two Methods of Nitsche. The methods use the bilinear form

$$
B_{h}(\phi, \psi)=a(\phi, \psi)-\left\langle\phi, \frac{\partial \psi}{\partial \nu}\right\rangle-\left\langle\frac{\partial \phi}{\partial \nu}, \psi\right\rangle+\beta h^{-1}\langle\phi, \psi\rangle,
$$

where $\langle\cdot, \cdot\rangle$ denotes the inner product in $L^{2}(\partial \Omega), \partial / \partial \nu$ the conormal derivative on $\partial \Omega$ and $\beta$ a positive constant. The functions in $S_{h}$ are not required to vanish on $\partial \Omega$. If $S_{h} \subset H^{1}$ with the restrictions to $\partial \Omega$ in $H^{1}(\partial \Omega)$, and if $S_{h}$ satisfies

$$
\begin{aligned}
& \inf _{\chi \in S_{h}}\left\{\|w-\chi\|+h\|w-\chi\|_{1}+h^{1 / 2}\|w-\chi\|_{L^{2}(\partial \Omega)}+h^{3 / 2}\|w-\chi\|_{H^{1}(\partial \Omega)}\right\} \leqslant C h^{s}\|w\|_{s}, \\
& 2 \leqslant s \leqslant r
\end{aligned}
$$


and an "inverse" assumption

$$
\left\|\frac{\partial \chi}{\partial \nu}\right\|_{L^{2}(\partial \Omega)} \leqslant C h^{-1 / 2}\|\chi\|_{H^{1}} \text { for } \chi \in S_{h}
$$

then it can be shown that $B_{h}$ is positive definite on $S_{h}$ for $\beta$ sufficiently large. The operators $T_{h}: L^{2} \rightarrow S_{h}$ are defined by

$$
B_{h}\left(T_{h} f, \chi\right)=(f, \chi) \text { for } \chi \in S_{h} .
$$

If in addition to the above assumptions,

$$
\|\chi\|_{L^{2}(\partial \Omega)} \leqslant C_{0} h^{1 / 2}\|\chi\|_{1} \text { for } \chi \in S_{h},
$$

where $C_{0}$ is sufficiently small, then $B_{h}$ is positive definite on $S_{h}$ even with $\beta=0$.

(3) The Lagrange Multiplier Method of Babuška. Let $\left\{\mathscr{T}_{h}\right\}$ be a family of subspaces of $H^{1}$ which satisfies

$$
\inf _{\chi \in \mathscr{T}_{h}}\left\{\|w-\chi\|+h\|w-\chi\|_{1}\right\} \leqslant C h^{s}\|w\|_{s} \quad \text { for } 1 \leqslant s \leqslant r,
$$

and let $\left\{\mathscr{T}_{h}^{\prime}\right\}$ be a family of subspaces of $H^{1}(\partial \Omega)$ such that

$$
\begin{array}{r}
\inf _{\chi^{\prime} \in \mathscr{T}_{h}^{\prime}}\left\{h^{-1 / 2}\left\|w^{\prime}-\chi^{\prime}\right\|_{H^{-1 / 2}(\partial \Omega)}+h^{1 / 2}\left\|w^{\prime}-\chi^{\prime}\right\|_{H^{1 / 2}(\partial \Omega)}\right\} \\
\leqslant C h^{s}\left\|w^{\prime}\right\|_{H^{s}(\partial \Omega)} \quad \text { for } \frac{1}{2} \leqslant s \leqslant r-\frac{3}{2} .
\end{array}
$$

Assume also the inverse property

$$
\left\|\chi^{\prime}\right\|_{H^{1}(\partial \Omega)} \leqslant C h^{-1}\left\|\chi^{\prime}\right\|_{L^{2}(\partial \Omega)} \text { for } \chi^{\prime} \in \mathscr{T}_{h}^{\prime} .
$$

With $\delta$ sufficiently small the family $\left\{S_{h}\right\}$ is defined by

$$
S_{h}=\left\{\chi \in \mathscr{T}_{\delta h},\left\langle\chi, \chi^{\prime}\right\rangle=0 \text { for all } \chi^{\prime} \in \mathscr{T}_{h}^{\prime}\right\} .
$$

It can be shown that the bilinear form $a(\cdot, \cdot)$ is positive definite on $S_{h} . T_{h}: L^{2} \rightarrow S_{h}$ is defined by

$$
a\left(T_{h} f, \chi\right)=(f, \chi) \text { for } \chi \in S_{h} .
$$

These methods satisfy the following three properties:

(i) $T_{h}$ is selfadjoint, positive semidefinite on $L^{2}$, and positive definite on $S_{h}$.

(ii) If $j$ is an integer which is greater than or equal to 0 , then there exists a constant $C(j)$ such that

$$
\left\|\left(T^{(j)}-T_{h}^{(j)}\right) f\right\| \leqslant C(j) h^{s}\|f\|_{s-2} \text { for } 2 \leqslant s \leqslant r .
$$

(iii) Define $L_{h}$ to be $T_{h}^{-1}$ on $S_{h}$. If $j$ is a nonnegative integer, then there exists a constant $C(j)$ which is independent of $h$ such that for $s, t \in[0, \tau]$

$$
\left|\left(L_{h}^{(j)}(t) \phi, \phi\right)\right| \leqslant C(j)\left(L_{h}(s) \phi, \phi\right) \quad \text { for all } \phi \in S_{h} .
$$

The proof of (i) is given in Section 8 of Bramble, Schatz, Thomée and Wahlbin [6]. The proof of property (ii) is in Theorem 5.1 of Sammon [21].

The following lemma contains a proof of (ii) for the "standard" Galerkin method.

LEMma 7.1. For $f \in L^{2}$, let $T_{h} f$ be defined by the "standard" Galerkin method, i.e., $S_{h} \in H_{0}^{1}$ and

$$
a\left(T_{h} f, \chi\right)=(f, \chi) \text { for all } \chi \in S_{h} .
$$


Then, for $2 \leqslant s \leqslant r$,

$$
\left\|\left(T^{(j)}-T_{h}^{(j)}\right) f\right\|_{1} \leqslant C(j) h^{s-1}\|f\|_{s-2}
$$

and

$$
\left\|\left(T^{(j)}-T_{h}^{(j)}\right) f\right\| \leqslant C(j) h^{s}\|f\|_{s-2} .
$$

Proof. The proof is by induction on $j$. The estimates $\left\|\left(T-T_{h}\right) f\right\|_{1} \leqslant C h^{s-1}\|f\|_{s-2}$ and $\left\|\left(T-T_{h}\right) f\right\| \leqslant C h^{s}\|f\|_{s-2}$ are well known. Let $e=\left(T_{h}-T\right) f$ and $e^{(j)}=$ $\left(T_{h}^{(j)}-T^{(j)}\right) f$. It follows from (7.1) that $a(e, \chi)=0$ for all $\chi \in S_{h}$. Therefore,

$$
\sum_{j=0}^{m}\left(\begin{array}{c}
m \\
j
\end{array}\right) a^{(m-j)}\left(e^{(j)}, \chi\right)=0 \quad \text { for all } \chi \in S_{h} \text {. }
$$

Since

$$
\begin{aligned}
\left\|e^{(m)}\right\|_{1}^{2} \leqslant & C a\left(e^{(m)}, e^{(m)}\right) \\
= & C\left(a\left(e^{(m)}, e^{(m)}-\chi\right)\right. \\
& \left.\quad+\sum_{j=0}^{m-1}\left(\begin{array}{c}
m \\
j
\end{array}\right)\left[a^{(m-j)}\left(e^{(j)}, e^{(m)}-\chi\right)-a^{(m-j)}\left(e^{(j)}, e^{(m)}\right)\right]\right), \\
\leqslant & \frac{1}{2}\left\|e^{(m)}\right\|_{1}^{2}+C\left(\sum_{j=0}^{m-1}\left\|e^{(j)}\right\|_{1}^{2}+\left\|e^{(m)}-\chi\right\|_{1}^{2}\right)
\end{aligned}
$$

for any $\chi \in S_{h}$, it follows by induction and the approximation property that

$$
\left\|e^{(m)}\right\|_{1} \leqslant C(m) h^{s-1}\|f\|_{s-2} .
$$

This is (7.2). To prove (7.3) note that for any $\phi \in L^{2}$ and $\chi \in S_{h}$,

$$
\begin{aligned}
\left(e^{(m)}, \phi\right) & =\left(e^{(m)}, L T \phi\right)=a\left(e^{(m)}, T \phi\right) \\
& =\sum_{j=0}^{m}\left(\begin{array}{c}
m \\
j
\end{array}\right) a^{(m-j)}\left(e^{(j)}, T \phi-\chi\right)-\sum_{j=0}^{m-1}\left(\begin{array}{c}
m \\
j
\end{array}\right)\left(e^{(j)}, L^{(m-j)} T \phi\right) \\
& \leqslant C\left(\sum_{j=0}^{m}\left\|e^{(j)}\right\|_{1}\right) \inf _{\chi \in S_{h}}\|T \phi-\chi\|_{1}+C \sum_{j=0}^{m-1}\left\|e^{(j)}\right\|\|T \phi\|_{2} .
\end{aligned}
$$

(7.3) follows by induction using (7.2) and the approximation property.

The next lemma applies to any method for which $T_{h}$ is symmetric and which satisfies (ii) and an inverse property.

LEMMA 7.2. If $T_{h}$ is symmetric and satisfies (ii) and the inverse property

(iv) $\left(L_{h} \phi, \phi\right)^{1 / 2} \leqslant C h^{-1}\|\phi\|$ for all $\phi \in S_{h}$,

then for $s, t \in[0, \tau]$ and integers $l, m \geqslant 0$,

$$
\left\|L_{h}^{(l)}(s) T_{h}^{(m)}(t) \phi\right\| \leqslant C(l, m)\|\phi\|
$$

and

$$
\left\|T_{h}^{(l)}(t) L_{h}^{(m)}(s) \phi\right\| \leqslant C(l, m)\|\phi\|,
$$

where $C(l, m)$ is independent of $h$. 
Proof. It follows from (iv) that $\left\|L_{h} \psi\right\| \leqslant C h^{-2}\|\psi\|$ for all $\psi \in S_{h}$. Since

$$
\begin{aligned}
L_{h}(s) T_{h}^{(m)} & (t)=L_{h}(s) T_{h}^{(m)}(t)-P L(s) T^{(m)}(t)+P L(s) T^{(m)}(t) \\
= & L_{h}(s) P\left(T_{h}^{(m)}(t)-T^{(m)}(t)\right)+L_{h}(s) P\left(T(s)-T_{h}(s)\right) L(s) T^{(m)}(t) \\
& +P L(s) T^{(m)}(t),
\end{aligned}
$$

property (ii) (with $s=2$ ), property (iv) and the triangle inequality imply that

$$
\left\|L_{h}(s) T_{h}^{(m)}(t) \phi\right\| \leqslant C\|\phi\| \text { for all } \phi \in S_{h} .
$$

This is (7.4) with $l=0$.

Also, since

$$
\left\|T_{h}^{(l)}(t) L_{h}(s) \phi\right\|=\sup _{\psi \in L^{2}} \frac{\left(T_{h}^{(l)}(t) L_{h}(s) \phi, \psi\right)}{\|\psi\|}=\sup _{\psi \in L^{2}} \frac{\left(\phi, L_{h}(s) T_{h}^{(l)}(t) \psi\right)}{\|\psi\|}
$$

it follows from (7.6) that

$$
\left\|T_{h}^{(l)}(t) L_{h}(s) \phi\right\| \leqslant C\|\phi\| \quad \text { for all } \phi \in S_{h} .
$$

This is (7.5) with $m=0$. The remaining parts of the lemma are proved using (7.6) and (7.7). For example, since

$$
L_{h}^{(1)}(s) T_{h}(t)=-\left(L_{h}(s) T_{h}^{(1)}(s)\right)\left(L_{h}(s) T_{h}(t)\right)
$$

and

$$
L_{h}^{(m)}(s) T_{h}(t)=-\sum_{j=1}^{m}\left(\begin{array}{c}
m \\
j
\end{array}\right)\left(L_{h}^{(m-j)}(s) T_{h}(t)\right)\left(L_{h}(t) T_{h}^{(j)}(s)\right)\left(L_{h}(s) T_{h}(t)\right),
$$

it follows by induction on $m$ (using (7.6) and (7.7)) that

$$
\left\|L_{h}^{(m)}(s) T_{h}(t) \phi\right\| \leqslant C\|\phi\| \quad \text { for all } \phi \in S_{h} .
$$

Similarly, the estimate

$$
\left\|T_{h}(t) L_{h}^{(m)}(s) \phi\right\| \leqslant C\|\phi\| \quad \text { for all } \phi \in S_{h},
$$

can be proved. Finally, the identities

$$
L_{h}^{(l)}(s) T_{h}^{(m)}(t)=\left(L_{h}^{(l)}(s) T_{h}(t)\right)\left(L_{h}(t) T_{h}^{(m)}(t)\right)
$$

and

$$
T_{h}^{(l)}(t) L_{h}^{(m)}(s)=\left(T_{h}^{(l)}(t) L_{h}(s)\right)\left(T_{h}(s) L_{h}^{(m)}(s)\right)
$$

used with (7.6), (7.7), (7.8) and (7.9) complete the proof of the lemma.

Acknowledgement. The author would like to thank Professor J. H. Bramble, Professor A. H. Schatz and Professor L. B. Wahlbin for their advice and inspiration.

Department of Mathematics

University of Tennessee

Knoxville, Tennessee 37996-1300

1. G. A. BAKER, "Error estimates for finite element methods for second order hyperbolic equations." SIAM J. Numer. Anal., v. 13, 1976, pp. 564-576.

2. G. A. Baker \& J. H. Bramble, "Semidiscrete and single step fully discrete approximations for second order hyperbolic equations," RAIRO Anal. Numér., v. 13, 1979, pp. 75-100.

3. G. A. BaKer, J. H. Bramble \& V. Thomet, "Single step Galerkin approximations for parabolic problems," Math. Comp., v. 31, 1977, pp. 818-847. 
4. G. A. Baker, V. A. Dougalis \& S. M. SeRbin, "High order accurate two-step approximations for hyperbolic equations," RAIRO Anal. Numér., v. 13, 1979, pp. 201-206.

5. J. H. Bramble \& P. H. Sammon, "Efficient higher order single step methods for parabolic problems: Part I," Math. Comp., v. 35, 1980, pp. 655-677.

6. J. H. Bramble, A. H. Schatz, V. ThOmEe \& L. B. Wahlbin, "Some convergence estimates for semidiscrete Galerkin type approximations for parabolic equations," SIAM J. Numer. Anal., v. 14, 1977, pp. 218-241.

7. M. Crouzerx, Sur l'Approximation des Équations Différentielles Opérationnelles Linéaires par des Méthodes de Runge-Kutta, Thèse, Université de Paris VI, 1975.

8. V. A. Dougalis, "Multistep Galerkin methods for hyperbolic equations," Math. Comp., v. 33, 1979, pp. 563-584.

9. V. A. Dougalis \& S. M. SeRBIN, “Two-step high order accurate full discretizations of second order hyperbolic equations," Proc. 3rd IMACS Symposium, Advances in Computer Methods for Partial Differential Equations (R. Vichnevetsky and R. S. Stepleman, eds.), IMACS, 1979, pp. 214-220.

10. J. Douglas, JR., T. DuPONT \& R. E. EwING, "Incomplete iteration for time-stepping a Galerkin method for a quasilinear parabolic problem," SIAM J. Numer. Anal., v. 16, 1979, pp. 503-522.

11. T. DUPONT, " $L^{2}$-estimates for Galerkin methods for second order hyperbolic equations," SIAM J. Numer. Anal., v. 10, 1973, pp. 880-889.

12. E. GEKELER, "Linear multistep methods and Galerkin procedures for initial boundary value problems," SIAM J. Numer. Anal., v. 13, 1976, pp. 536-548.

13. E. GeKELER, "Galerkin-Runge-Kutta methods and hyperbolic initial boundary value problems," Computing, v. 18, 1977, pp. 79-88.

14. G. GiLARDI, "Teoremi di regolarità per la soluzione di un'equazione differenziale astratta lineare del secondo ordine," Istit. Lombardo Accad. Sci. Lett. Rend. A, v. 106, 1972, pp. 641-675.

15. L. A. Hageman \& D. M. Young, Applied Iterative Methods, Academic Press, New York, 1981.

16. J. D. LAMBerT, Computational Methods in Ordinary Differential Equations, Wiley, New York, 1973.

17. J. L. Lions, E. Magenes, Nonhomogeneous Boundary Value Problems and Applications, Vol. I, Springer-Verlag, Berlin and New York, 1972.

18. J. L. Lions \& E. MAGenes, Nonhomogeneous Boundary Value Problems and Applications, Vol. II, Springer-Verlag, Berlin and New York, 1972.

19. H. Mingyou \& V. ThOMEE, "Some convergence estimates for semidiscrete type schemes for time-dependent nonselfadjoint parabolic equations," Math. Comp., v. 37, 1981, pp. 327-346.

20. P. H. Sammon, Approximations for Parabolic Equations with Time-Dependent Coefficients, Ph.D. Thesis, Cornell University, 1978.

21. P. H. SAmmon, "Convergence estimates for semidiscrete parabolic equation approximations," SIAM J. Numer. Anal., v. 19, 1982, pp. 68-92.

22. M. Zlámal, "Finite element multistep discretizations of parabolic boundary value problems," Math. Comp., v. 29, 1975, pp. 350-359. 